山्山FRANÇAISE

$>\mathrm{DE}$

$\stackrel{1}{\simeq}$ PÉDAGOGIE

\section{Revue française de pédagogie}

Recherches en éducation

$196 \mid 2016$

Apprendre à lire et à écrire au cours préparatoire : enseignements d'une recherche collective

\title{
Lire et écrire au cours préparatoire : nouvelles perspectives pour la mesure du temps scolaire et de ses effets
}

Reading and writing in first-grade: New perspectives for the allocation of school time and its effects

Céline Piquée et Caroline Viriot-Goeldel

\section{(2) OpenEdition}

Journals

Édition électronique

URL : https://journals.openedition.org/rfp/5072

DOI : 10.4000/rfp.5072

ISSN : 2105-2913

Éditeur

ENS Éditions

Édition imprimée

Date de publication : 30 septembre 2016

Pagination : $23-48$

ISSN : 0556-7807

\section{Référence électronique}

Céline Piquée et Caroline Viriot-Goeldel, « Lire et écrire au cours préparatoire : nouvelles perspectives pour la mesure du temps scolaire et de ses effets », Revue française de pédagogie [En ligne], 196 | 2016, mis en ligne le 30 septembre 2019, consulté le 27 janvier 2022. URL : http://journals.openedition.org/ rfp/5072 ; DOI : https://doi.org/10.4000/rfp.5072 


\title{
Lire et écrire au cours préparatoire : nouvelles perspectives pour la mesure du temps scolaire et de ses effets
}

\author{
Céline Piquée \\ Caroline Viriot-Goeldel
}

\begin{abstract}
Cet article analyse la distribution du temps consacré à l'enseignement de la lecture-écriture à l'école primaire et ses effets sur les acquis des élèves. Basé sur une enquête empirique auprès de 2507 élèves de 131 classes de cours préparatoire, il examine les différentes dimensions de cet enseignement : la phonographie, la lecture, la compréhension, l'écriture, l'étude de la langue. Les résultats confirment la grande variabilité du temps entre les classes. Ils mettent en évidence les effets du temps alloué par l'enseignant aux différentes composantes étudiées, effets particulièrement visibles pour les élèves initialement les plus faibles. Les modalités d'organisation du temps scolaire sont également examinées, révélant l'importance de la présence de l'enseignant auprès des élèves les plus faibles.
\end{abstract}

Mots-clés (TESE) : temps d'enseignement, lecture, progrès scolaire, enseignement primaire

\section{Introduction}

Les controverses sur les méthodes d'enseignement de la lecture demeurent vives et récurrentes dans notre pays. Elles prennent appui sur des discours sur les pratiques et sur des études fondées sur des données déclaratives. Largement médiatisées, ces études mobilisent généralement des descriptions globales en termes de «méthodes» (voir par exemple Deauvieau, 2013; Ministère de l'Éducation nationale [MEN], 1999). La présente étude vise à dépasser les «déclarations» en termes de «méthodes» pour s'intéresser à la des- cription et à l'efficacité de pratiques d'enseignement de la lecture-écriture effectivement observées dans le détail des tâches données aux élèves.

Nombre d'études expérimentales conduites ces dernières décennies ont permis d'avancer dans la connaissance de l'apprentissage de la lecture-écriture et de son enseignement (voir les synthèses d'Ecalle \& Magnan, 2015; de Kamil, Pearson, Birr Moje et al., 2011 ; et du National Reading Panel, 2000). Reste néanmoins à mesurer l'efficacité des pratiques effectives des enseignants dans les classes, en contexte et dans toute leur complexité. Qu'en est-il de l'efficacité des 
pratiques validées par la recherche expérimentale lorsque les enseignants construisent eux-mêmes leurs séances, sans mettre en œuvre un protocole élaboré par un chercheur? Ces effets sont-ils les mêmes lorsque les enseignants font interagir les différentes dimensions du lire-écrire que les études expérimentales visent à isoler? Observe-t-on les mêmes effets des pratiques sur le temps long que sur les quelques semaines ou quelques mois d'une étude expérimentale? Ces quelques questions parmi beaucoup d'autres laissent apparaître combien ces études ponctuelles d'un phénomène isolé ne peuvent prétendre appréhender tout ce que signifie «s'engager dans, apprendre de ou enseigner avec une diversité de textes dans une diversité de classes» (Kamil, Pearson, Birr Moje et al., 2011). Elles soulignent la pertinence d'un passage du « what works? » à « what works, for whom, when, why and how?» en intégrant une pluralité de dimensions et de méthodologies complémentaires (Kamil, Pearson, Birr Moje et al., 2011).

À cet égard, la mesure des temps d'enseignement et d'apprentissage offre une perspective intéressante. Une quantification des pratiques observées en classe en unités de temps permet en effet de mettre en relation une variété de pratiques enseignantes avec les progrès effectués par les élèves de ces classes, afin de mesurer l'efficacité des pratiques dans la lignée des travaux sur les effets-maîtres conduits à l'étranger et en France depuis la Beginning Teacher Evaluation Studies (Denham \& Lieberman, 1980). Elle permet par exemple d'établir dans quelle mesure les élèves bénéficiant d'un temps d'enseignement important consacré à un apprentissage améliorent leurs performances dans le domaine concerné. Elle rend également possible la détermination de seuils, en deçà ou au-delà desquels les élèves ne progressent pas suffisamment. Berliner (1990) considère ainsi le temps d'instruction comme une variable particulièrement productive de la recherche en éducation, permettant de comprendre, de prédire et de réguler l'enseignement. Ce courant de recherche prend appui sur la relation exposée par Carroll (1963), selon laquelle l'apprentissage des élèves serait fonction du rapport entre le temps d'apprentissage offert aux élèves et celui dont les élèves auraient besoin pour apprendre.

La question est alors de savoir comment mesurer ce temps d'enseignement. De nombreuses recherches sur le temps scolaire se réfèrent au modèle de Smyth (1985) pour situer les différents niveaux d'analyse du
2012; Delhaxhe, 1997; Suchaut, 1996; Suchaut, Bougnères \& Bouguen, 2014).

Le premier niveau de ce modèle se limite au temps d'enseignement officiellement prescrit. Le deuxième se centre sur la quantité d'instruction proposée à l'élève, déduction faite notamment des absences des enseignants et des élèves, ainsi que des sorties scolaires. Le troisième niveau du modèle examine le temps effectivement alloué au contenu des activités scolaires, les occasions d'apprendre. Le quatrième niveau de ce modèle articule la dimension quantitative du temps alloué par l'enseignant avec la dimension qualitative du temps d'engagement de l'élève dans la tâche. II renvoie ainsi au temps durant lequel l'élève consacre son attention à la tâche proposée. Le cinquième niveau enfin correspond au temps d'apprentissage académique (ALT pour Academic Learning Time), c'est-à-dire «au temps durant lequel l'élève s'est impliqué dans une tâche d'apprentissage dont les objectifs coïncident avec des items de l'épreuve d'évaluation et dont le degré de difficulté permet à l'élève de produire un maximum (90\%) de bonnes réponses» (Delhaxhe, 1997, p. 114). Ce cinquième niveau est ainsi d'une nature différente des précédents, dans la mesure où le temps n'y est plus appréhendé comme une simple provision de ressource. Dans la lignée des travaux de psychologie américains de Carroll et de Bloom (Chopin, 2010), I'ALT est centré sur l'adéquation entre les caractéristiques et besoins de l'élève d'une part, et les enseignements qui lui sont proposés d'autre part. On y trouve ainsi des items liés à la difficulté de la tâche, à la clarté de la consigne, au rythme de travail, etc.

\section{Le temps scolaire dans les recherches antérieures}

D'une manière générale, les recherches sur l'effet-maître soulignent l'importance de la gestion de ce temps scolaire, l'enseignant efficace étant aussi celui qui maximise ce temps (Bressoux, 1994). De nombreux travaux se situant au niveau 3 du modèle de Smyth ont pu mettre en évidence une relation significative entre les temps d'enseignement et la réussite des élèves (voir Suchaut, 1996 et Chopin, 2010 pour une synthèse). Le constat vaut également pour l'enseignement de la lecture en langue française (Suchaut, 1996; Suchaut, Bougnères \& Bouguen, 2014). Ces études révèlent toutes la variabilité de l'usage du temps scolaire (Bressoux, Amigues, Arnoux et al., 2002), soulignant de fait l'écart entre curriculum prescrit et curriculum réel (Perrenoud, 
1984). Ainsi, au cours préparatoire, Suchaut (1996) montre que le temps hebdomadaire consacré à la lecture varie entre 7,4 heures et 15,6 heures et que moins de $10 \%$ des enseignants respectent les horaires prescrits par les textes officiels. Ces différences dans l'allocation de temps entre les disciplines sont très faiblement expliquées par les caractéristiques classiques du contexte scolaire telles que la taille de la classe, sa tonalité sociale ou encore son degré d'hétérogénéité; l'enseignant serait donc l'arbitre principal dans ce domaine. Cette relation entre le temps d'enseignement proposé par le maître et les progrès des élèves n'est néanmoins ni systématique, ni toujours linéaire. Par exemple, Suchaut (1996) signale qu'au cours préparatoire l'augmentation du temps consacré à la lecture engendre une amélioration des acquis des élèves dans ce domaine mais avec des effets de seuil qui varient en fonction du niveau initial des élèves : au-delà de 11 heures hebdomadaires, l'augmentation du temps n'exerce plus d'effet pour les élèves forts, au-delà de 13 heures pour les élèves faibles; il existe donc bien un effet différencié du temps alloué sur les progressions. La relation dépend en fait à la fois des disciplines, des caractéris- tiques des élèves, de la qualité de l'enseignement, du fait que ce temps soit consacré à des pratiques didactiques pertinentes, qu'il soit adapté aux élèves et en adéquation avec leurs besoins (Berliner, 1990).

Par ailleurs, on conçoit aisément combien il peut être vain de consacrer du temps à l'enseignement d'une compétence si les élèves n'y prêtent pas attention ou si d'autres tâches d'exécution prennent le pas sur l'engagement cognitif attendu. C'est pourquoi un quatrième niveau de mesure du temps prend en compte l'engagement des élèves. "Le temps que l'élève consacre à l'appropriation d'une compétence $a$, d'évidence, un lien plus étroit avec l'apprentissage que les temps d'enseignement alloués par le maître » (Crahay, 2012, p. 254). L'engagement des élèves constitue ainsi une dimension supplémentaire à articuler avec l'étude du temps alloué aux apprentissages. Autrement dit, la question du temps d'instruction n'est pas tant celle du temps d'enseignement offert aux élèves que celle du temps durant lequel les élèves sont engagés dans un enseignement de qualité adéquat.

La question de l'engagement des élèves dans les tâches appelle donc un renouvellement de la mesure 
du temps scolaire (Chopin, 2010; Suchaut, Bougnères \& Bouguen, 2014) tant du point de vue conceptuel que du point de vue méthodologique.

\section{La mesure du temps scolaire : à la recherche d'un compromis}

Le choix du niveau de mesure du temps dans le cadre de cette publication' se situe principalement au niveau du temps effectivement alloué par l'enseignant aux différents domaines du lire-écrire, soit au niveau 3 du modèle de Smyth. Nous pensons en effet que, dans l'apprentissage de la lecture-écriture, tout n'est pas de la seule responsabilité de l'élève qui parvient ou pas à s'engager dans les tâches proposées par l'enseignant (Bressoux, Amigues, Arnoux et al., 2002; Chopin, 2010). Les choix méthodologiques opérés nous permettent néanmoins d'atteindre un degré de précision supérieur à celui de nombreuses autres études de ce type, nous situant au-delà du niveau 3 du modèle de Smyth et de l'usage qui en a, jusqu'à présent, été fait.

Tout d'abord, plusieurs recherches consacrées à l'étude du temps alloué par le maître prennent en compte le temps d'enseignement déclaré par le maître (Chopin, 2010; Crahay, 2012). Dans la présente étude, la mesure du temps résulte d'observations chronométrées de séances de classes pour plus d'exactitude.

Ensuite, la plupart des études de ce type mesurent des durées globales, telles que par exemple le temps total consacré à la lecture ou aux mathématiques. Là aussi, notre étude propose un grain d'analyse plus fin, détaillant le temps passéà travailler les différentes composantes du lire-écrire : la phonographie, la lecture, la compréhension, l'écriture, l'étude de la langue. Chaque composante est elle-même subdivisée en différentes rubriques. Par exemple, la phonographie se compose des rubriques "étudier les phonèmes", "étudier les syllabes », «étudier les lettres», etc. La liste des composantes et de leur rubrique est proposée en annexe. Ces distinctions permettent alors l'analyse des choix didactiques des enseignants dans l'enseignement de la lecture-écriture, apportant ainsi un souffle nouveau aux recherches sur les occasions d'apprendre.

Ensuite encore, afin de dépasser une mesure au niveau des séances prévues dans l'emploi du temps

1 Cette publication est issue de la recherche «Lire-Écrire. Étude de l'influence des pratiques d'enseignement de la lecture et de l'écriture sur la qualité des premiers apprentissages» (Goigoux, des enseignants (Bressoux, Amigues, Arnoux et al., 2002), notre étude se place au niveau des tâches prescrites aux élèves. Avec Sensevy (2007, p. 351), nous partageons en effet l'idée que «la tâche donnée aux élèves constitue ainsi, pour la recherche préoccupée de la compréhension de l'efficacité professorale, une unité fondamentale de l'analyse ». À mi-chemin entre les niveaux 3 et 4 du modèle de Smyth (1985), la mesure du temps scolaire retenue renvoie alors à une unité de découpage du temps scolaire qui est celui des tâches, unités temporelles caractérisant ce que le professeur demande à ses élèves de faire. Nous définissons une tâche en fonction du but que l'enseignant assigne à ses élèves dans des conditions déterminées (Goigoux, 2002). En d'autres termes, il s'agit de décrire les occasions d'apprendre que chaque enseignant rend possibles à travers les tâches qu'il propose à ses élèves.

Enfin la mesure retenue permet de s'approcher du temps d'engagement des élèves (niveau 4), car les temps morts (temps consacrés à d'autres dimensions que la lecture-écriture, comme par exemple à l'organisation matérielle, à des interruptions diverses, à la gestion de la classe, à l'attente d'un groupe d'élèves ou de la classe entière, etc.) ont été déduits du temps alloué aux apprentissages du lire-écrire. Or ces temps ont été définis par la littérature comme des exemples archétypaux de non-engagement (Berliner, 1990; Rosenshine, cité par Crahay, 2012), ceux-là mêmes qui ont été déduits de la Beginning Teachers Study, étude de référence sur le temps d'engagement des élèves ayant donné lieu à l'élaboration du concept d'Academic Learning Time. De la même manière, aux côtés d'une déduction des temps individuels d'inattention des élèves, c'est au niveau 4 du modèle présenté plus haut que Smyth (1985) déduit les temps de non-engagement collectifs (Chopin, 2010). Ainsi, si nous ne prétendons pas mesurer individuellement le temps d'engagement de chaque élève, opération délicate d'un point de vue méthodologique lorsque les échantillons sont de taille conséquente, nous avons cherché à nous en approcher à l'instar de plusieurs études ayant appréhendé à la fois le temps et l'engagement des élèves (Taylor, Pearson, Clark et al., 2000; Taylor, Pearson, Peterson et al., 2003).

\section{Types de temps et rendements différenciés}

Certaines études suggèrent que tous les temps ne suscitent pas des rendements équivalents. Ainsi, les élèves 
seraient plus engagés dans la tâche lors du travail collectif de la classe avec l'enseignant, que lors du travail individuel. Ce dernier serait en effet plus propice aux distractions et intrusions, ou encore à la perte de concentration (Rosenshine, 1980). S'agissant du travail individuel, on observe également des différences liées à l'investissement de l'enseignant (Bressoux, Amigues, Arnoux et al., 2002). Si celui-ci circule dans les rangs et interagit avec les élèves, leur engagement dans la tâche est plus important que dans le cas contraire, et le rendement de ce temps d'activité est alors plus élevé (Berliner, 1990). Ces travaux suscitent donc des questions sur les effets de l'organisation pédagogique associée aux temps alloués aux tâches de lecture-écriture, en particulier sur la présence/absence de l'enseignant comme condition de réalisation des tâches.

"La question du lien entre temps et enseignement rebondit et se répète en empruntant toujours de nouvelles figures» (Chopin, 2010, p. 2). Cet article propose ainsi de nouvelles perspectives pour l'étude du temps alloué aux différentes composantes du lire-écrire, dépassant les limites les plus fréquentes de ce type de recherche. II se situe au plus près de la proposition d'activité faite aux élèves, dans une perspective didactique, tout en intégrant un certain nombre de dimensions liées aux modalités de travail. Après avoir précisé les aspects méthodologiques de la recherche, nous nous intéresserons dans un premier temps au temps total d'enseignement du lire-écrire et à ses effets sur les performances des élèves de cours préparatoire. Puis un deuxième temps examinera plus précisément les temps consacrés aux différents domaines de la lecture-écriture. Une dernière section sera consacrée aux effets du temps alloué aux tâches de lecture-écriture, assorti de ses conditions de réalisation : en présence de l'enseignant ou en autonomie. Tout au long de ces trois types d'analyse, nous interrogerons le caractère différencié de ces effets, en fonction du niveau initial des élèves et du niveau moyen des classes. Les résultats seront alors discutés, au regard des travaux antérieurs sur le temps scolaire mais également des débats actuels sur l'enseignement de la lecture-écriture.

\section{Méthodologie}

\section{Participants}

Les analyses portent sur un échantillon de 131 classes de cours préparatoire $(\mathrm{CP})$ réparties dans 13 académies françaises. Ces classes ne sont pas strictement repré- sentatives de la situation nationale car elles ont été choisies pour présenter, dans leur ensemble, une variété de situations pédagogiques et didactiques, variété nécessaire à l'examen des effets de ces situations sur les performances des élèves (Duru-Bellat \& Mingat, 1998). Parmi ces classes, 29,8\% relèvent de l'éducation prioritaire, $20,6 \%$ ont un fonctionnement hebdomadaire réparti sur 4,5 journées. L'ancienneté moyenne des enseignants est de 16,4 ans (écarttype $=7,4$ ), et de 8,4 ans au CP (écart-type $=5,3$ ).

Dans ces classes, 2507 élèves ont participé à l'enquête. On compte $50,3 \%$ de filles et $4,2 \%$ d'élèves en retard scolaire. $71,7 \%$ des élèves parlent uniquement le français à la maison contre $6,7 \%$ qui ne le parlent pas. Les autres $21,6 \%$ parlent au moins deux langues à la maison, dont le français. Le milieu social d'origine a été défini à partir de la profession la plus élevée des deux parents : 19,5\% appartiennent à un milieu favorisé (parent cadre), 28,1\% à un milieu intermédiaire (parent agriculteur exploitant, artisan, commerçant ou profession intermédiaire) et 51,4\% à un milieu défavorisé (employé, ouvrier, retraité ou inactif).

\section{Instruments de mesure des performances des élèves}

Les performances des élèves ont été évaluées en début d'année de CP ( $2^{\mathrm{e}}$ semaine de septembre 2013 ) dans les domaines du code (analyse phonologique, connaissance du nom des lettres, lecture de mots familiers et de pseudomots), de la compréhension (vocabulaire, phrases et textes entendus) et de l'écriture (prénom, mots, phrases courtes). Ils ont ensuite été à nouveau évalués en fin d'année (juin 2014) dans les domaines du code (analyse phonologique, lecture de mots familiers et de pseudomots, fluence), de la compréhension (phrases et textes en lecture autonome, textes entendus) et de l'écriture (dictée, production de texte, copie). Le score global, initial et final, repose sur la somme de chaque sous-score standardisé. Les indices de consistance interne (analyse de fiabilité par le coefficient de Cronbach) sont supérieurs à 0,80 , indiquant par là une construction cohérente des épreuves dans chacun des domaines considérés.

\section{Mesure du temps scolaire}

Pour parvenir à homogénéiser les découpages temporels et pour affecter chaque tâche à une catégorie préétablie, nous avons élaboré une typologie jointe en 
annexe. Cette typologie est structurée en trente et un types de tâches, eux-mêmes regroupés en cinq grands ensembles correspondant aux domaines d'enseignement du lire-écrire à l'école (MEN, 2008, 2015) : phonographie, lecture, compréhension, écriture et étude de la langue. Treize d'entre eux exigent de coder aussi la nature de l'unité linguistique sur laquelle ils portaient (lettre, syllabe, mot, phrase ou texte) afin de distinguer, par exemple, la dictée de syllabes de la dictée de phrases. Au final, si dans cet article le temps sera appréhendé aux deux premiers niveaux uniquement, la typologie élaborée permet d'analyser l'offre d'enseignement à quatre niveaux, de plus en plus fins :

- celui du budget-temps total (hors temps morts);

- celui des ensembles de tâches (5 catégories);

- celui des tâches (31 catégories);

- celui des tâches par type d'unité linguistique (73 catégories).

Les temps morts qui ont été soustraits des tâches de lecture-écriture sont les épisodes de plus d'une minute qui ne sont pas dédiés à l'enseignement de la lecture ou de l'écriture: appel des élèves qui déjeunent à la cantine, période d'attente lorsqu'une partie au moins des élèves a terminé son travail, phase de relaxation, distribution de matériel, découpage d'étiquettes, collage de photocopies... Chaque tâche a été ainsi chronométrée et codée au moyen de la typologie. Un changement de tâche est repéré par l'enquêteur chaque fois que l'enseignant donne un nouveau but à ses élèves ou modifie les conditions pour l'atteindre.

\section{Procédures}

Les observations se sont déroulées simultanément dans toutes les classes pendant trois semaines complètes, une par trimestre en novembre 2013 (S1), mars (S2) et mai 2014 (S3). Cet échantillonnage temporel nous semble pouvoir révéler les caractéristiques des pratiques ordinaires des maîtres dans la mesure où chacun s'était engagé à ne rien changer de sa pratique habituelle et à nous prévenir en cas d'événement exceptionnel (par exemple une sortie scolaire) afin de prévoir une observation supplémentaire.

Pour reconstituer le budget-temps d'une classe, chaque enquêteur a assisté à toutes les séances de lecture-écriture proposées aux élèves lors des trois semaines d'observation, y compris dans des séances à dominante disciplinaire autre que le français lorsque l'enseignant lui indiquait préalablement y avoir inclus (enseignants-chercheurs assistés de docteurs et doctorants, de conseillers pédagogiques et maîtres formateurs en activité ou retraités) préalablement formés se référaient à un cahier des charges d'une soixantaine de pages et déposaient le résultat de leurs observations à la fin de chaque semaine sur un site web dédié et protégé. Nous renvoyons le lecteur à Goigoux, Jarlégan et Piquée (2015) pour une présentation détaillée du dispositif méthodologique.

Pour prévenir les difficultés du travail de catégorisation des tâches données aux élèves, une année de travail et de pré-test a été nécessaire afin de vérifier que toutes les tâches utilisées au CP étaient classables, de s'assurer de l'étendue raisonnable des catégories, de la pertinence des choix des exemples prototypiques (voir en annexe) et de l'homogénéité du codage. Outre la formation des enquêteurs via des entraînements basés sur des vidéos de séances de classes et des catégories de tâches définies de manière suffisamment ample pour limiter les doutes, ces exemples prototypiques permettaient de lever ceux qui persistaient. Enfin, un contrôle de ce travail de catégorisation a été conduit a posteriori à partir des vidéos de classes (Riou, 2017). 36 séances ont été à nouveau codées et, lorsque l'on se situe au niveau des grandes composantes du lire-écrire (phonographie, lecture, compréhension...), le rapport entre les temps mesurés en classe et ceux mesurés a posteriori est supérieur à 0,80 (Riou, 2017, p. 204).

\section{Méthode d'analyse des données}

Les données ont été analysées à l'aide de modèles de régression multiniveaux sous le logiciel SPSS, version 20. Les modèles multiniveaux sont appropriés à notre questionnement puisque les données présentent une structure hiérarchisée et emboîtée (Bressoux, 2007) : les élèves (niveau 1) sont scolarisés dans des classes (niveau 2). Avant d'examiner les effets des variables cibles (ici le temps consacré aux tâches), il est d'usage de procéder dans un premier temps à une décomposition de la variance totale des performances finales des élèves selon les différents niveaux (la variance intraclasse et la variance interclasse). Cette décomposition est présentée dans un modèle dit «vide» (voir tableau 1 en annexe). Dans un deuxième temps, il s'agit d'expliquer chaque partie de la variance par des variables de même niveau (c'est-à-dire par des variables de niveau 1 pour la variance intraclasse et par des variables de niveau 2 pour la variance interclasses; voir tableau 1, modèles 1 et 2 , en annexe). 
Tableau 2. Moyenne et dispersion du temps hebdomadaire consacré aux tâches de lecture et écriture

\begin{tabular}{l|c|c|c|c}
\hline Variable & Moyenne & Écart-type & Minimum & Maximum \\
\hline $\begin{array}{l}\text { Temps total consacré au lire-écrire } \\
\text { mesuré en novembre (semaine 1) }\end{array}$ & $7 \mathrm{~h} 30 \mathrm{~min}$ & $1 \mathrm{~h} 35 \mathrm{~min}$ & $3 \mathrm{~h} 43 \mathrm{~min}$ & $13 \mathrm{~h} 11 \mathrm{~min}$ \\
\hline $\begin{array}{l}\text { Temps total consacré au lire-écrire } \\
\text { mesuré en mars (semaine 2) }\end{array}$ & $7 \mathrm{~h} 26 \mathrm{~min}$ & $1 \mathrm{~h} 33 \mathrm{~min}$ & $4 \mathrm{~h} 20 \mathrm{~min}$ & $12 \mathrm{~h} 41 \mathrm{~min}$ \\
\hline $\begin{array}{l}\text { Temps total consacré au lire-écrire } \\
\text { mesuré en mai (semaine 3) }\end{array}$ & $7 \mathrm{~h} 9 \mathrm{~min}$ & $1 \mathrm{~h} 47 \mathrm{~min}$ & $3 \mathrm{~h} 32 \mathrm{~min}$ & $17 \mathrm{~h} 4 \mathrm{~min}$ \\
\hline $\begin{array}{l}\text { Temps moyen hebdomadaire } \\
\text { consacré au lire-écrire (Budgetot) }\end{array}$ & $\mathbf{7 h ~} \mathbf{2 2} \mathrm{min}$ & $1 \mathrm{~h} 25 \mathrm{~min}$ & $\mathbf{4 h ~} 19 \mathrm{~min}$ & $13 \mathrm{~h} 14 \mathrm{~min}$ \\
\hline
\end{tabular}

Note $: \mathrm{N}=131$.

Au niveau 1, les variables utilisées pour ces analyses préalables sont celles habituellement contrôlées dans les analyses multivariées:

- le niveau de l'élève en début de $C P$, préalablement centré et réduit $(m=0 ; s=1)$;

- son sexe;

- son milieu social;

- son âge;

- la langue parlée à la maison.

Les données présentées dans le tableau 1 en annexe indiquent que ces variables agissent ici de manière tout à fait classique (Mingat, 1991). Plus le niveau de l'élève est élevé en début d'année, plus il l'est en fin d'année. Les filles, les élèves de milieu favorisé, nés en début d'année civile et parlant au moins deux langues, dont le français, à la maison, affichent de meilleures progressions au cours de leur année de CP.

Au niveau classe, les variables utilisées pour ces analyses préalables sont :

- I'organisation du temps hebdomadaire (4 jours versus 4,5 jours);

- l'appartenance éventuelle à un réseau d'éducation prioritaire;

- le niveau moyen initial de la classe, obtenu par agrégation des scores individuels (la moyenne des scores des élèves par classe a ensuite été centrée et réduite $(m=0$ et $s=1)$;

- I'hétérogénéité moyenne initiale de la classe, mesurée par l'écart-type associé au niveau initial moyen des classes;

- la tonalité sociale de la classe, mesurée par le pourcentage d'élèves favorisés;

- I'ancienneté de l'enseignant au CP.

Le choix des variables de contrôle de niveau 2 s'est opéré après plusieurs spécifications, dans le but de ne retenir que celles dont l'impact était significatif. Or, selon les variables cibles testées dans les modèles, le niveau de significativité de chaque variable de contrôle est susceptible d'évoluer. Ainsi, si certaines de ces variables n'exercent pas d'effet significatif dans le cadre des analyses conduites dans cet article, nous avons choisi de les conserver afin d'assurer la comparabilité des analyses qui seraient produites sur d'autres sujets à partir des mêmes données.

\section{Résultats}

\section{Le temps consacré à l'enseignement de la lecture-écriture}

\section{Description}

Les données recueillies permettent d'estimer le temps total qu'ont consacré les enseignants aux tâches de lecture et d'écriture, en excluant les temps morts. Le tableau 2 en donne une première vision.

Les classes de l'échantillon consacrent en moyenne 7 h 22 min hebdomadaires à l'enseignement du lireécrire au $C P$, avec un écart-type moyen de près d'1 h30 min Les valeurs minimum et maximum indiquent qu'entre deux classes, le temps consacré au lire-écrire peut passer du simple au triple. On constate une relative stabilité du temps selon les périodes d'observation : les écarts entre les temps mesurés en semaine 1 et 2 ne sont pas statistiquement significatifs alors que la diminution, somme toute modeste, relevée en période 3 l'est ( $T=2,376$, au seuil de 0,019 par rapport à la semaine 2). On précisera également la corrélation significative (au seuil de 0,000) entre les temps mesurés à chaque période, qui signifie que plus le temps consacré à l'enseignement du lire-écrire est élevé lors de la semaine 1, plus il l'est lors des semaines 2 et 3 . 
Figure 2. Dispersion du temps total (répartition par déciles en minutes)

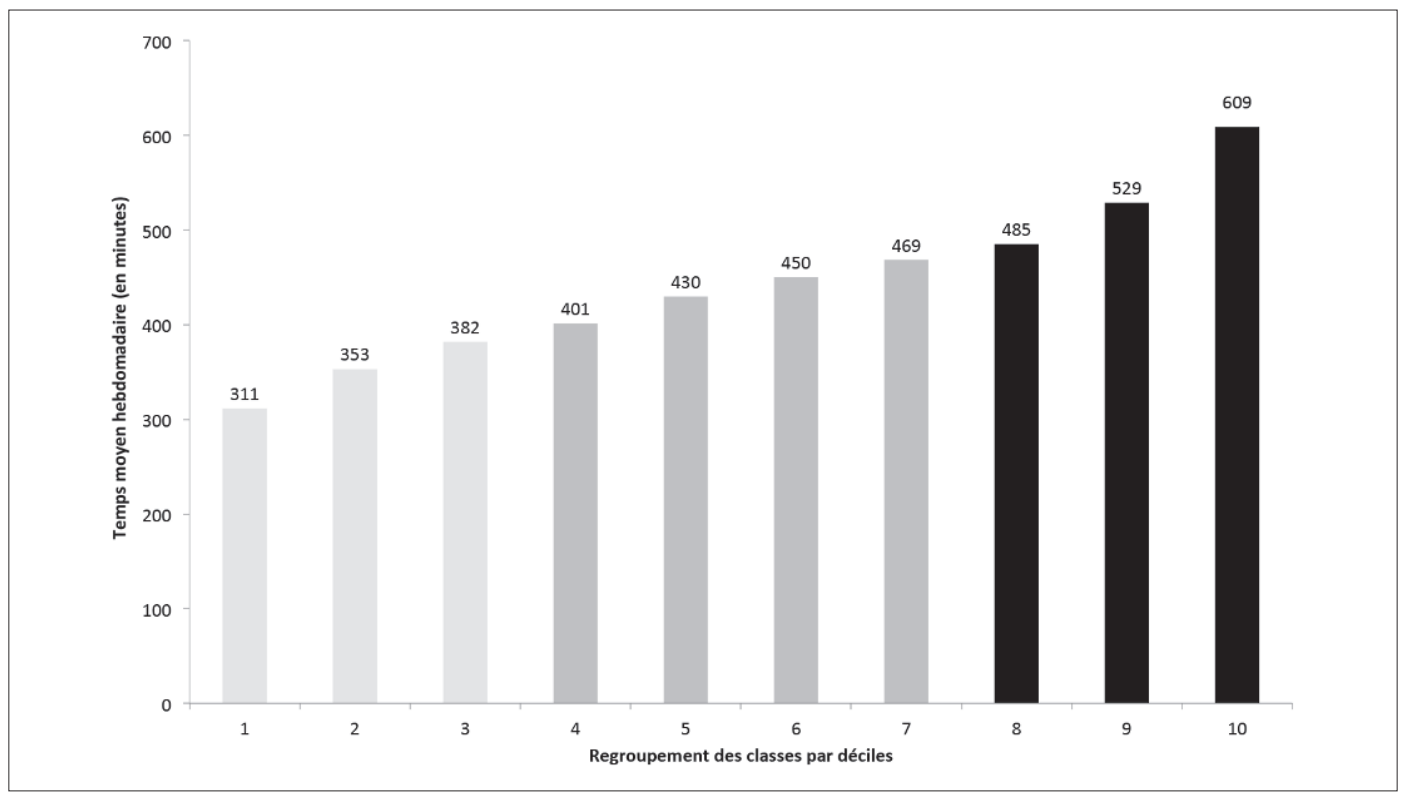

Lecture : les classes du premier décile consacrent au maximum 311 minutes hebdomadaires aux tâches d'enseignement du lire-écrire.

L'examen des déciles permet d'appréhender plus précisément la dispersion de la distribution du temps dans les 131 classes de l'échantillon (voir figure 2).

Le premier décile regroupe les $10 \%$ des classes qui consacrent le moins de temps aux activités de lireécrire (en moyenne 311 min hebdomadaires, soit 5 h $11 \mathrm{~min}$ ). Le dernier décile regroupe les $10 \%$ des classes qui consacrent le plus de temps aux activités de lire-écrire (en moyenne $609 \mathrm{~min}$, soit $10 \mathrm{~h} 9 \mathrm{~min}$ ). Entre ces deux extrêmes, le temps consacré aux tâches de lecture-écriture varie donc du simple au double.

\section{Effets sur le score global des élèves en fin de cours préparatoire}

Le temps total consacré aux tâches de lecture-écriture (variable $=$ zbudgetot $^{2}$ ) a fait l'objet de modélisations ayant pour objectif d'examiner si, toutes choses égales par ailleurs, les variations constatées entre les classes entraînent des variations sur le score global des

2 Les variables indépendantes relatives aux différents temps examinés résultent d'abord de la somme des temps observés pendant les trois semaines d'observation. Ces temps ont ensuite été centrés réduits (moyenne $=0$; écart-type $=1$ ) pour faciliter l'interprétation des résultats. Chaque nom de variable est alors précédé élèves à la fin du CP (voir tableau 3). Le modèle 1 introduit la variable zbudgetot uniquement. Ce modèle vise à identifier un effet moyen linéaire sur les performances finales des élèves. Le modèle 2 examine la possibilité de la non-linéarité de la relation (Bressoux, 2010). C'est ici une forme quadratique (en U ou U inversé) qui est testée afin de déceler d'éventuels effets de seuil. Les modèles 3 et 4 cherchent à préciser si les effets de la variable cible s'exercent dans certaines conditions plus particulièrement : les effets sont-ils différents selon le niveau initial des élèves (modèle 3), selon le niveau moyen de la classe en début d'année (modèle 4) ?

À la lecture du tableau 3 , on constate que le temps total alloué au lire-écrire n'exerce aucun effet moyen linéaire (modèle 1), non linéaire (modèle 2) ou différencié en fonction du niveau initial des élèves (modèle 3 ). Dit autrement, ce temps n'impacte pas les progrès des élèves en général, ni à partir d'un certain seuil, ni certains élèves uniquement, les plus forts ou les plus faibles notamment. Toutefois, on relève que ce temps exerce des effets différenciés selon le niveau moyen des classes en début d'année scolaire (modèle 4) : plus le niveau initial des classes est faible, plus l'augmentation du temps exerce des effets positifs. Des estimations portant sur des sous-échantillons (la moitié des 
Tableau 3. Effets du temps total sur le score global en fin de CP

\begin{tabular}{|c|c|c|c|c|}
\hline Paramètres & Modèle 1 & Modèle 2 & Modèle 3 & Modèle 4 \\
\hline \multicolumn{5}{|l|}{ Effets fixes } \\
\hline Constante & $-0,03(0,22)$ & $-0,09(0,16)$ & $-0,09(0,16)$ & $-0,06(0,16)$ \\
\hline Score individuel initial & $0,71(0,02)^{* * *}$ & $0,71(0,02)^{* * *}$ & $0,72(0,02)^{* * *}$ & $0,71(0,02)^{* * *}$ \\
\hline Fille (référence garçon) & $0,08(0,03)^{* * *}$ & $0,08(0,03)^{* * *}$ & $0,08(0,03)^{* * *}$ & $0,08(0,03)^{* * *}$ \\
\hline \multicolumn{5}{|l|}{ Profession des parents: } \\
\hline Catégorie défavorisée & $-0,11(0,04)^{* * *}$ & $-0,11(0,04)^{* * *}$ & $-0,11(0,04)^{* * *}$ & $-0,11(0,04)^{* * *}$ \\
\hline $\begin{array}{l}\text { Catégorie intermédiaire } \\
\text { (référence catégorie favorisée) }\end{array}$ & $-0,03(0,04)$ & $-0,03(0,04)$ & $-0,03(0,04)$ & $-0,03(0,04)$ \\
\hline \multicolumn{5}{|l|}{ Âge : } \\
\hline Né avant 2007 (retard scolaire) & $-0,61(0,07)^{* * *}$ & $-0,61(0,07)^{* * *}$ & $-0,61(0,07)^{* * *}$ & $-0,61(0,07)^{* * *}$ \\
\hline Né entre mai et août 2007 & $-0,02(0,03)$ & $-0,02(0,03)$ & $-0,02(0,03)$ & $-0,02(0,03)$ \\
\hline $\begin{array}{l}\text { Né entre sept. et déc. } 2007 \\
\text { (référence né entre janv. et avril } \\
2007 \text { ou en avance) }\end{array}$ & $-0,02(0,03)$ & $-0,02(0,03)$ & $-0,02(0,03)$ & $-0,02(0,03)$ \\
\hline \multicolumn{5}{|l|}{ Langue parlée à la maison: } \\
\hline Autre & $0,03(0,06)$ & $0,03(0,06)$ & $0,03(0,06)$ & $0,03(0,06)$ \\
\hline $\begin{array}{l}\text { Français + autre } \\
\text { (référence français) }\end{array}$ & $0,12(0,04)^{* * *}$ & $0,12(0,04)^{* * *}$ & $0,12(0,04)^{* * *}$ & $0,12(0,04)^{* * *}$ \\
\hline $\begin{array}{l}\text { Semaine de } 4 \text { jours } \\
\text { (référence } 4,5 \text { jours) }\end{array}$ & $-0,07(0,07)$ & $0,07(0,07)$ & $-0,08(0,07)$ & $-0,08(0,07)$ \\
\hline Éducation prioritaire & $-0,03(0,07)$ & $-0,04(0,07)$ & $-0,04(0,07)$ & $-0,05(0,07)$ \\
\hline Niveau moyen initial & $-0,20(0,08)^{* *}$ & $-0,20(0,08)^{* *}$ & $-0,20(0,08)^{* *}$ & $-0,19(0,08)^{* *}$ \\
\hline Hétéro moyenne initiale & $0,05(0,15)$ & $0,05(0,16)$ & $0,05(0,15)$ & $0,03(0,15)$ \\
\hline \% d'élèves de milieu favorisé & $0,26(0,19)$ & $0,25(0,19)$ & $0,26(0,18)$ & $0,28(0,18)$ \\
\hline Ancienneté au CP & $0,01(0,01)$ & $0,01(0,01)$ & $0,01(0,01)$ & $0,01(0,01)$ \\
\hline zbudgetot & $0,04(0,03)$ & $0,04(0,03)$ & $0,04(0,03)$ & $0,05(0,03)^{* *}$ \\
\hline zbudgetot*zbudgetot & & $0,01(0,01)$ & & \\
\hline zbudgetot*sco. ind. ini. & & & $-0,01(0,01)$ & \\
\hline zbudgetot*niveau moy. ini. & & & & $-0,19(0,07)^{* * *}$ \\
\hline \multicolumn{5}{|l|}{ Effets aléatoires } \\
\hline Niveau 2 : var. interclasses & $0,0719(0,01)$ & $0,0718(0,01)$ & $0,0711(0,01)$ & $0,0666(0,01)$ \\
\hline Niveau 1 : var. intraclasse & $0,3898(0,01)$ & $0,3898(0,01)$ & $0,3898(0,01)$ & $0,3898(0,01)$ \\
\hline$-2 \log L$ & 4898,564 & 4898,456 & 4897,384 & 4890,585 \\
\hline
\end{tabular}

Notes : N=2 481 (26 élèves ont été retirés des analyses pour cause de données manquantes relatives à leur âge ou la profession de leurs parents). Seuil de significativité des effets fixes : ${ }^{*}$ : significatif au seuil de $10 \%$; ${ }^{*}$ : significatif au seuil de $5 \%$; $^{* * *}$ : significatif au seuil de $1 \%$. Les erreurs type des coefficients figurent entre parenthèses. 
Tableau 4. Moyenne horaire hebdomadaire et dispersion des cinq domaines de tâches

\begin{tabular}{l|c|c|c|c}
\hline Variables (domaines) & $\begin{array}{c}\text { Durée moyenne } \\
\text { hebdomadaire }\end{array}$ & Écart-type & $\begin{array}{c}\text { Coefficient de } \\
\text { variation }\end{array}$ & $\begin{array}{c}\% \text { budget total } \\
\text { du lire-écrire }\end{array}$ \\
\hline PG (phonographie) & $1 \mathrm{~h} 21 \mathrm{~min}$ & $29 \mathrm{~min}$ & $35,8 \%$ & $18 \%$ \\
\hline L (lecture) & $1 \mathrm{~h} 42 \mathrm{~min}$ & $31 \mathrm{~min}$ & $30,4 \%$ & $23 \%$ \\
\hline C (compréhension) & $1 \mathrm{~h} 8 \mathrm{~min}$ & $24 \mathrm{~min}$ & $35,3 \%$ & $16 \%$ \\
\hline E (écriture) & $2 \mathrm{~h} 23 \mathrm{~min}$ & $40 \mathrm{~min}$ & $28,0 \%$ & $32 \%$ \\
\hline EL (étude de la langue) & $39 \mathrm{~min}$ & $30 \mathrm{~min}$ & $76,9 \%$ & $9 \%$ \\
\hline
\end{tabular}

Notes : $N=131$. Le coefficient de variation est le rapport de l'écart-type à la moyenne, généralement exprimé en pourcentage. Il permet une mesure de l'intensité de la dispersion d'une distribution indépendamment de la valeur absolue de la moyenne considérée, rendant ainsi plus lisibles les comparaisons entre variables.

classes les plus faibles d'un côté, la moitié des classes les plus fortes de l'autre) permettent de préciser que seuls les élèves des classes initialement les plus faibles bénéficient d'une augmentation du temps consacré à la lecture-écriture.

\section{Le temps consacré aux cinq domaines du lire-écrire}

\section{Description}

Le temps total accordé au lire-écrire au CP a été décomposé en 5 domaines de tâches dont la distribution est présentée dans le tableau 4.

Ce sont les tâches d'écriture qui occupent le plus de temps, avec une moyenne hebdomadaire de $2 \mathrm{~h} 23 \mathrm{~min}$ (un tiers du temps total). Elles sont suivies par les tâches de lecture (en moyenne $1 \mathrm{~h} 42$ min hebdomadaires, soit environ un quart du temps total). Les tâches de phonographie et celles de compréhension comptent pour un peu moins de $20 \%$ du temps total chacune, celles d'étude de la langue moins de $10 \%$. Les coefficients de variation indiquent que ce sont les tâches de lecture et d'écriture qui font l'objet des plus faibles variations entre les classes. Une variété un peu plus forte est constatée pour les tâches de phonographie et de compréhension. Mais ce sont clairement celles d'étude de la langue, les moins fréquentes, qui différencient le plus les classes les unes des autres. Au-delà de ce temps moyen hebdomadaire, la figure 3 permet de visualiser l'évolution du temps consacré à chacun de ces cinq domaines au cours de l'année.

Alors que le temps total consacré au lire-écrire (tous domaines confondus) est relativement stable selon les périodes de l'année, les temps consacrés à chaque domaine à l'intérieur du temps total subissent
Les tâches de phonographie sont celles qui diminuent le plus fortement au cours de l'année (les écarts entre chaque période sont statistiquement significatifs au seuil de 0,000 ), passant de près de $2 \mathrm{~h}$ en semaine 1 à un peu plus d' $1 \mathrm{~h}$ en semaine 3 . Les tâches d'écriture et de lecture diminuent elles aussi au cours de l'année, significativement entre la première et la deuxième semaine d'observation pour l'écriture et entre la deuxième et la troisième pour la lecture. À l'inverse, les tâches de compréhension et d'étude de la langue augmentent significativement entre la semaine 1 et la semaine 2 pour se stabiliser ensuite. Ces variations ne sont pas surprenantes au regard de l'évolution de l'enseignement attendue en cours d'année, en fonction des compétences des élèves et des objectifs prioritaires que se donne l'enseignant.

\section{Effets sur le score global des élèves en fin de cours préparatoire}

Les modélisations intégrant les temps consacrés à chacun de ces groupes de tâches (voir tableau 5 en annexe) révèlent uniquement un effet moyen linéaire du temps consacré aux tâches d'étude de la langue : une unité de temps supplémentaire entraîne un gain de $11 \%$ d'écart-type sur le score final. Les variations constatées dans les autres domaines ne semblent pas, à ce stade des analyses, exercer d'effet moyen linéaire sur le score global final des élèves. La possibilité de phénomènes non linéaires a été testée et apparaît significative pour le temps consacré aux tâches de compréhension (voir figure 4 ; les détails du modèle sont donnés dans le tableau 6 en annexe).

La figure 4 indique que plus le temps consacré à des tâches de compréhension est élevé, plus les élèves progressent. Mais on observe également un point de rupture dans la courbe qui signifie qu'au-delà de ce 
Figure 3. Évolution du temps consacré aux 5 domaines de tâches au cours de l'année de CP

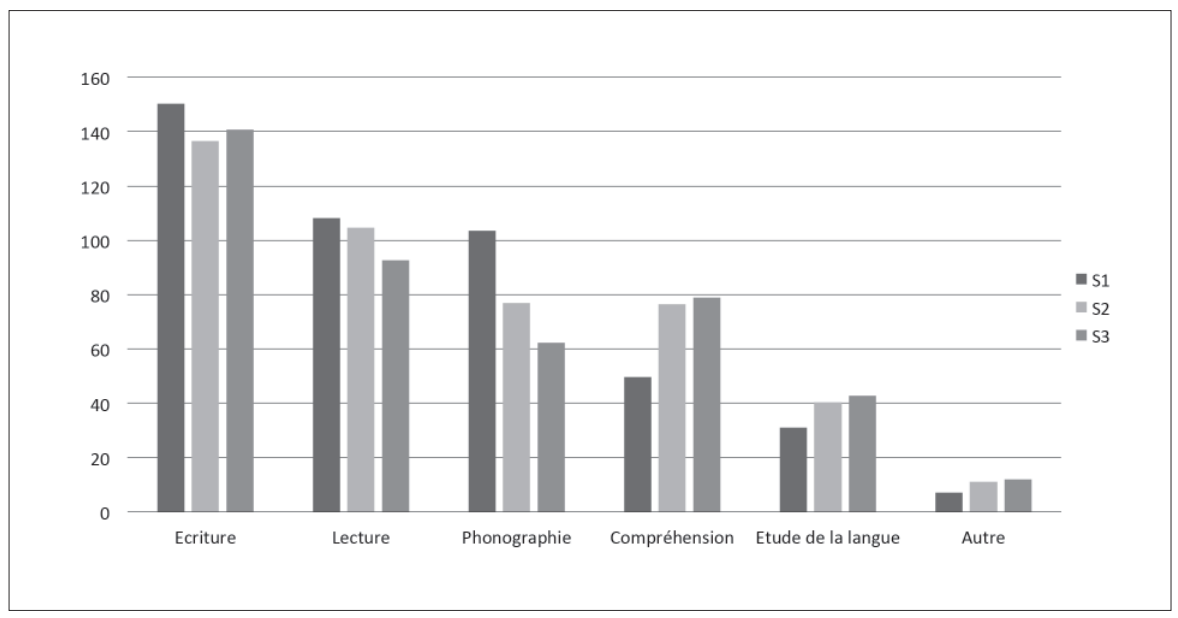

Lecture : lors de la première semaine d'observation (S1), les classes de l'échantillon ont consacré en moyenne 150 minutes à des tâches d'écriture.

Figure 4. Effet du temps consacré aux tâches de compréhension sur le score global final (en pourcentage d'écart-type)

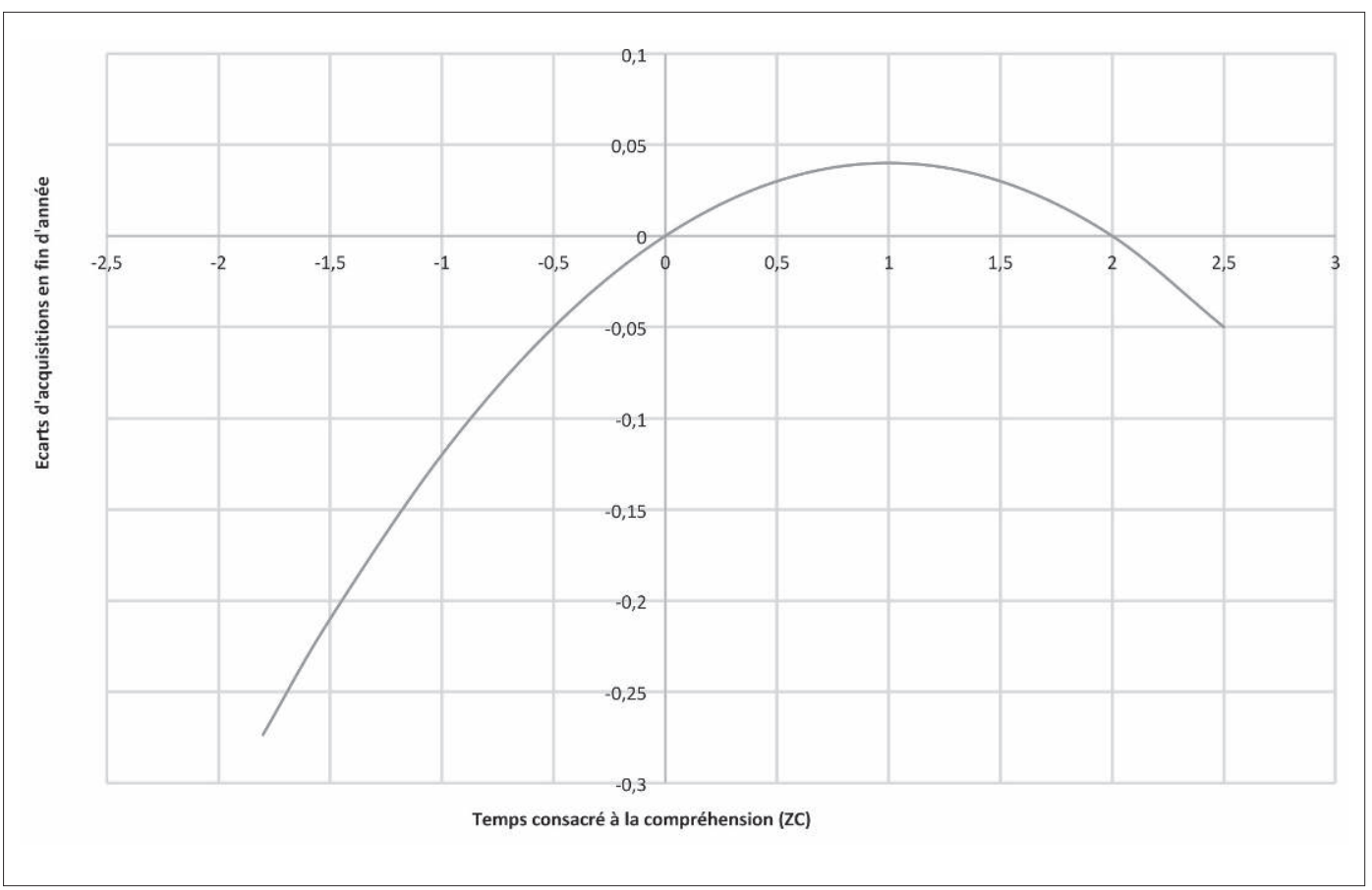


Figure 5. Effets du temps consacré à l'étude de la langue sur le score global final selon le niveau initial des élèves (en pourcentage d'écart-type)

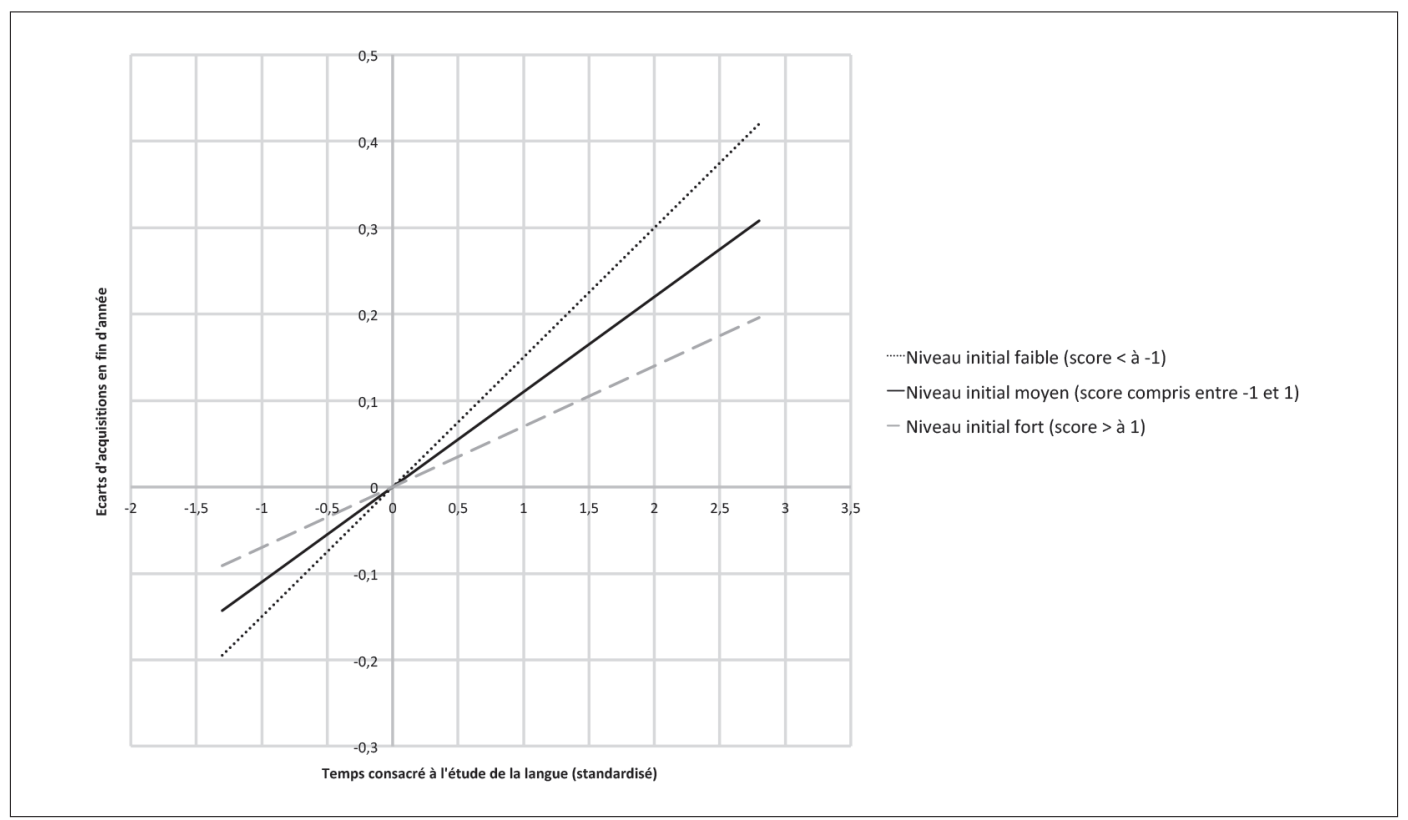

seuil (estimé à environ 1 h 40 min hebdomadaires), les gains pour les élèves ralentissent. Par ailleurs, la partie droite de la figure montre qu'entre les classes dans lesquelles les tâches de compréhension sont les plus fréquentes et les classes dans lesquelles ces tâches sont moyennement fréquentes, les écarts d'acquisitions entre élèves sont plutôt faibles. En revanche, lorsque le temps consacré aux tâches de compréhension est relativement faible (cadran bas gauche), alors les écarts entre élèves sont plus forts : on estime à environ $20 \%$ d'écart-type l'écart d'acquisitions entre des élèves qui s'y consacreraient à peine une demiheure par semaine (valeur -1,5 de ZC) et des élèves qui $s^{\prime} y$ consacreraient un peu plus d' $1 \mathrm{~h}$ (valeur 0 de ZC).

Par ailleurs, les effets du temps consacré à la compréhension s'avèrent également variables selon le niveau moyen initial des classes (voir tableau 6 en annexe). Des spécifications statistiques ont été réalisées sur des sous-échantillons de classes (la moitié des classes les plus faibles d'un côté, la moitié des classes les plus fortes de l'autre). Elles indiquent que c'est seulement dans les classes dont le niveau moyen est le plus faible que l'augmentation du temps consacré aux tâches de compréhension exerce des effets positifs linéaires sur le score global final des élèves, c'est-à-dire sans qu'ap-
Enfin, des effets différenciés selon le niveau initial des élèves sont observés pour le temps consacré à l'étude de la langue (voir tableau 6 en annexe). Ces effets peuvent être visualisés dans la figure 5 : plus les élèves sont faibles et plus la pente de la droite est forte, indiquant par là un effet d'autant plus fort que les élèves sont initialement faibles.

\section{Temps consacré au lire-écrire et modalités de travail}

\section{Description}

Les données recueillies permettent également de reconstituer le temps que les élèves ont consacré aux tâches de lecture-écriture en présence de l'enseignant. On constate que les élèves passent en moyenne 6 h 30 min hebdomadaires (écart-type $=1$ h $30 \mathrm{~min}$ ) sur des tâches en présence de leur enseignant. Par soustraction, les élèves passent donc en moyenne un peu moins d'1 h par semaine sur des tâches de lecture-écriture en dehors de la présence de l'enseignant titulaire, c'est-à-dire soit dans un groupe de travail autonome, en moyenne 43 min hebdomadaires (écarttype = $36 \mathrm{~min}$ ), soit avec un maître supplémentaire, en moyenne $8 \mathrm{~min}$ hebdomadaires (écart-type = $13 \mathrm{~min}$ ). Le temps passé avec un maître supplémentaire est en 
Tableau 7. Moyenne hebdomadaire et dispersion des cinq domaines de tâches selon les modalités de travail

\begin{tabular}{l|c|c|c}
\hline \multicolumn{4}{|c}{ Durée moyenne hebdomadaire (écart-type) } \\
\hline & En présence de l'enseignant & Avec un enseignant supplémentaire & En autonomie \\
\hline Phonographie (PG) & $1 \mathrm{~h} 11 \mathrm{~min}(27 \mathrm{~min})$ & $2 \mathrm{~min}(4 \mathrm{~min})$ & $8 \mathrm{~min}(10 \mathrm{~min})$ \\
Lecture (L) & $1 \mathrm{~h} 31 \mathrm{~min}(29 \mathrm{~min})$ & $2 \mathrm{~min}(4 \mathrm{~min})$ & $8 \mathrm{~min}(10 \mathrm{~min})$ \\
Compréhension (C) & $1 \mathrm{~h}(28 \mathrm{~min})$ & $1 \mathrm{~min}(3 \mathrm{~min})$ & $8 \mathrm{~min}(10 \mathrm{~min})$ \\
Écriture (E) & $2 \mathrm{~h} 5 \mathrm{~min}(38 \mathrm{~min})$ & $2 \mathrm{~min}(6 \mathrm{~min})$ & $15 \mathrm{~min}(16 \mathrm{~min})$ \\
Etude de la langue (EL) & $35 \mathrm{~min}(23 \mathrm{~min})$ & $0,5 \mathrm{~min}(2 \mathrm{~min})$ & $2 \mathrm{~min}(4 \mathrm{~min})$ \\
\hline
\end{tabular}

Note $: \mathrm{N}=131$.

Tableau 8. Effets du temps passé avec le maître sur le score global en fin de CP (présentation synthétique)

\begin{tabular}{|c|c|c|c|c|}
\hline Paramètres & Modèle 1 & Modèle 2 & Modèle 3 & Modèle 4 \\
\hline \multicolumn{5}{|l|}{ Effets fixes } \\
\hline ztps & $0,06(0,03)^{* *}$ & $0,06(0,03)^{* *}$ & $0,06(0,03)^{* *}$ & $0,06(0,03)^{* *}$ \\
\hline ztps*ztps & & $-0,01(0,01)$ & & \\
\hline ztps* sco. ind. ini. & & & $-0,03(0,01)^{* *}$ & \\
\hline ztps* niveau moy. ini. & & & & $-0,15(0,07)^{* *}$ \\
\hline \multicolumn{5}{|l|}{ Effets aléatoires } \\
\hline Niveau 2 : var. interclasses & $0,0702(0,01)$ & $0,0700(0,01)$ & $0,0691(0,01)$ & $0,0669(0,01)$ \\
\hline Niveau 1 : var. intraclasse & $0,3898(0,01)$ & $0,3898(0,01)$ & $0,3892(0,01)$ & $0,3898(0,01)$ \\
\hline$-2 \log L$ & 4896,217 & 4895,995 & 4891,013 & 4891,317 \\
\hline
\end{tabular}

Notes: $\mathrm{N}=2481$. Seuil de significativité des effets fixes : ${ }^{*}$ : significatif au seuil de $10 \%$; ${ }^{* *}$ : significatif au seuil de $5 \%$; ${ }^{* * *}$ : significatif au seuil de $1 \%$. Les erreurs type des coefficients figurent entre parenthèses.

moyenne relativement faible puisque 25 enseignants seulement (soit moins de $20 \%$ de l'échantillon) ont déclaré que leur école bénéficiait d'un maître surnuméraire. Ces temps sont disponibles pour chaque groupe de tâches ainsi que présenté dans le tableau 7.

\section{Effets sur le score global des élèves en fin de cours préparatoire}

Le temps consacré aux tâches de lecture-écriture en présence de l'enseignant (variable $=$ ztps) a fait l'objet de différentes modélisations (voir tableau $8^{3}$ ), suivant la même organisation que celle présentée dans le tableau 3. Le modèle 1 introduit la variable ztps uniquement afin d'identifier un effet moyen linéaire sur

3 Par souci d'économie de lecture, nous choisissons une présentation synthétique des résultats sans reprise des effets des variables de contrôle de niveau 1 et niveau 2 (ces effets sont présentés dans le tableau 3 et ne subissent que d'infimes variations dans les modèles du tableau 8). les performances finales des élèves. Le modèle 2 examine l'hypothèse de non-linéarité de la relation. Les modèles 3 et 4 cherchent à préciser les effets de la variable cible en fonction du niveau initial des élèves (modèle 3) ou du niveau moyen de la classe en début d'année (modèle 4).

Les analyses révèlent qu'une unité supplémentaire de temps passé avec le maître entraîne une augmentation du score final équivalent à $6 \%$ de l'écart-type (voir modèle 1), sans qu'il semble exister de seuil (modèle 2). Ces effets sont différenciés selon le niveau initial des élèves (modèle 3 ) ou de la classe (modèle 4). Des analyses complémentaires indiquent que ces effets ne s'observent qu'auprès des élèves dont le score initial est inférieur à la moyenne et d'autant plus fortement que le niveau initial moyen des classes est faible.

Considéré sur les cinq groupes de tâches, seul le temps passé avec le maître sur des tâches d'étude de la langue exerce un effet moyen linéaire positif, cet effet s'élève à $11 \%$ d'écart-type (voir tableau 9 en 
annexe). Les effets d'interaction et les non-linéarités ont été modélisés (voir tableau 10 en annexe), révélant des effets similaires à ceux observés en considérant le temps global. Ainsi, les effets du temps avec le maître consacré aux tâches d'études de la langue sont d'autant plus forts que les élèves sont faibles en début d'année. Les effets du temps consacré à la compréhension rencontrent un extremum (estimé à environ $86 \mathrm{~min}$ ) et s'observent surtout dans les classes initialement les plus faibles. La prise en compte de la présence du maître révèle également des effets positifs auprès des élèves les plus faibles pour les tâches de lecture et dans les classes les plus faibles pour les tâches de phonographie.

Pour ce qui concerne les temps passés avec un maître supplémentaire ou en autonomie, nous avons vu précédemment que, considérés pour chaque domaine de tâches, ils s'avèrent très faibles. Les modélisations n'ont donc introduit ces temps que d'une façon globale, c'est-à-dire tous domaines confondus (voir tableau 11 en annexe). On observe alors qu'une augmentation du temps passé sans le maître conduit à une progression moindre, inférieure de $5 \%$ d'écarttype, et cet effet est d'autant plus fort que les élèves sont initialement faibles. L'augmentation du temps passé avec un maître supplémentaire exerce des effets positifs, en tendance, seulement auprès des élèves de bon niveau initial. Pour confirmer ce résultat, une dernière analyse a introduit dans les modélisations la présence d'un maître surnuméraire dans l'école. Les résultats affichent la même tendance, à savoir un effet positif visible uniquement auprès des élèves de bon niveau en début de CP.

\section{Discussion}

Cette recherche propose à une large échelle, 2507 élèves scolarisés dans 131 classes de $C P$, une mesure améliorée du temps scolaire et de ses effets. Elle situe en effet le temps scolaire au niveau des tâches données par le maître, excluant le plus précisément possible les temps d'inactivité des élèves. Si nous ne prétendons pas mesurer le temps d'engagement individuel de chaque élève dans les apprentissages, le temps consacré aux apprentissages relevant du lire-écrire est mesuré avec plus de précision que celle requise par le niveau 3 de Smyth, s'approchant ainsi du niveau 4. En effet, l'individualisation de la mesure au niveau $4 \mathrm{du}$ modèle de Smyth (1985) pose aux chercheurs un cer- difficulté d'observer en permanence le comportement de chacun des élèves d'une classe, rappelons avec Delhaxhe (1997) que les signes extérieurs d'engagement dans une tâche sont loin d'être totalement fiables.

À mi-chemin entre les niveaux 3 et 4 donc, nos choix de mesure nous permettent également d'éviter l'écueil principal de ce modèle lorsque la mesure du temps s'opère au niveau de l'élève (niveaux 4 et 5 du modèle). Le reproche formulé est celui d'une «réification» du temps indépendamment des choix pédagogiques et didactiques des enseignants, «comme si chaque élève était caractérisé par un temps d'apprentissage indépendant de toute dimension pédagogique préexistant à toute situation d'enseignement» (Chopin, 2010, p. 13).

La présente étude montre ainsi que sur les 24 heures hebdomadaires d'enseignement, 7 h 22 min sont en moyenne consacrées à des tâches de lecture-écriture. Considérant d'une part que les $24 \mathrm{~h}$ hebdomadaires prescrites comportent les temps de récréation, et d'autre part que nous avons soustrait tous les temps morts (distribution de cahier, temps d'attente, etc.), on peut estimer que la réalité des classes est relativement proche des prescriptions officielles de $10 \mathrm{~h}$ hebdomadaires (MEN, 2008). On observe néanmoins une grande variété entre les classes, ce temps pouvant varier du simple au double entre le premier et le dernier décile. L'étude permet également d'appréhender la répartition des temps d'enseignement entre les différents domaines du lire-écrire. Elle rend caduques les vives controverses sur l'enseignement du code phonographique (Dehaene, 2013), permettant d'établir que l'étude systématique du code phonographique est pratiquée dans toutes les classes.

Au-delà de la description, I'analyse du temps alloué aux différentes tâches de lecture-écriture permet de mettre en évidence les effets de certains choix didactiques opérés par le maître. À ce titre, cette recherche possède une dimension heuristique forte pour l'étude de l'effet-maître, et plus particulièrement de l'efficacité des pratiques. La mise en relation effectuée dans le cadre de cette étude entre le temps consacré aux différents apprentissages du lire-écrire et les progrès des élèves laisse apparaître que «la relation temps/apprentissage n'est ni aussi simple, ni aussi directe qu'il y paraît» (Aronson, Zimmerman \& Carlos, 1998). Si nous n'avons pas mis en évidence d'effet moyen linéaire du temps total d'enseignement sur les performances des élèves, des analyses plus détaillées ont en revanche permis de mettre au jour un effet différencié selon le niveau moyen des classes en début d'année. Les élèves des classes initialement les plus faibles bénéficient en effet 
d'une augmentation du temps consacré à l'enseignement de la lecture-écriture.

L'analyse des effets du temps consacré aux différents domaines du lire-écrire sur les performances des élèves met en évidence l'effet du temps consacré à l'étude de la langue sur les performances globales en lecture-écriture des élèves, effet d'autant plus important que les élèves sont initialement faibles. La catégorie "étude de la langue» comprenait à la fois des tâches consacrées à la morphologie, au lexique et à la syntaxe. Sanchez, Magnan et Ecalle (2012) ont montré l'influence des connaissances morphologiques d'élèves de grande section de maternelle sur l'apprentissage de la lecture au CP. De nombreuses études ont également établi que le niveau de vocabulaire des élèves exerçait des effets positifs sur les compétences en identification des mots et en compréhension en lecture, même si cette deuxième relation est essentiellement documentée à partir de la $2^{\mathrm{e}}$ année de l'école élémentaire (NICHD, 2005). De manière complémentaire à ces travaux menés au niveau "élève», notre étude souligne le rôle de l'école en la matière. Le développement de compétences en morphologie, lexique, syntaxe ne relève pas uniquement de dispositions acquises dans l'environnement familial. Cette étude révèle en effet le rôle des enseignants, à travers les choix qu'ils opèrent, car il s'agit clairement de choix au vu de la grande diversité des pratiques observées dans ce registre.

Dans le prolongement des travaux de Bianco et Bressoux (2009), qui montraient qu'un enseignement explicite de la compréhension en moyenne et en grande section de maternelle exerçait des effets jusqu'en $C P$, nos analyses montrent aussi que la réalisation de tâches de compréhension au cours du CP exerce des effets positifs sur les performances des élèves. Nous avons pu préciser que ces effets sont en particulier visibles dans les classes ayant un niveau moyen initialement faible. Ces résultats confirment notamment que l'enseignement de la compréhension, que les prescriptions officielles envisagent comme un continuum de la petite section au $C M 2$, ne saurait être mis entre parenthèses au $C P$, et ce en dépit de l'attention à consacrer à l'apprentissage de l'identification des mots durant cette année, comme le soulignaient déjà Storch et Whitehurst (2002).

La prise en compte des modalités de travail a permis de préciser encore ces résultats. Nous avons ainsi pu établir que le temps consacréà des tâches de lecture-écriture en présence de l'enseignant exerce des effets positifs sur les performances des élèves. Nos résultats rejoignent ainsi ceux d'études américaines plus anciennes souli- gnant une différence de qualité entre le temps de travail avec ou sans le maître (Berliner, 1990), différence à laquelle, dans notre étude, les élèves les plus faibles sont les plus sensibles. Les effets positifs de la présence d'un enseignant supplémentaire dans la classe seulement auprès des élèves initialement de bon niveau sont plus délicats à interpréter. Ils sont potentiellement liés à la répartition des tâches et des élèves opérée entre l'enseignant titulaire et l'enseignant supplémentaire. En Grande-Bretagne, les travaux de Blatchford, Russell et Webster (2012) ont montré que les écoles ont tendance à confier les élèves les plus faibles aux maîtres surnuméraires, permettant alors à l'enseignant titulaire de la classe de concentrer son action sur les élèves les meilleurs.

D'une manière générale, hormis dans le domaine de l'écriture, la plupart des effets mis en évidence sont des effets différenciés selon le niveau initial des élèves. Dans cet article, les effets des pratiques enseignantes concernent les élèves et/ou les classes les plus faibles, ou bien sont plus forts auprès de ce public, qu'il s'agisse du temps consacré à l'apprentissage ou encore de la présence du maître durant ce temps. Notre étude montre ainsi que les élèves de $C P$ les plus faibles sont plus sensibles aux pratiques d'enseignement de la lecture mises en œuvre par les maîtres, à l'instar d'autres travaux français et anglo-saxons (Bianco, Pellenq, Lambert et al., 2012; Connor, Morrison \& Slominski 2006; Connor, Piasta, Fishman et al., 2009). Ces derniers soulignent par conséquent la nécessité de prévenir activement l'apparition des difficultés d'apprentissage de la lecture auprès de ces élèves faibles à l'entrée de l'école élémentaire ou avant (Bianco, Pellenq, Lambert et al., 2012; Snow, Burns \& Griffin, 1998).

Dans cet article, l'échantillon de classes n'est pas strictement représentatif de la situation nationale. Même si les comparaisons avec les données ministérielles disponibles nous indiquent qu'il n'en est pas très éloigné, il convient de rester prudent quant aux généralisations que l'on souhaiterait émettre des résultats descriptifs. Par ailleurs, on pourra regretter le caractère un peu trop global des analyses. D'une part, les performances des élèves en français sont appréhendées de manière regroupée, mêlant les compétences en code, compréhension et écriture. D'autre part, les tâches confiées aux élèves ne sont appréhendées que du point de vue du temps et disent peu de choses des pratiques concrètes mises en œuvre dans les classes. Par exemple, le temps total consacré à la compréhension est construit sur la somme de tâches très diverses, que l'on peut suspecter d'exercer des influences différentes. En effet, 
Taylor et ses collaborateurs ont montré par exemple la supériorité des tâches de compréhension de haut niveau sur les tâches techniques routinisées (Taylor, Pearson, Clark et al., 2000). De même, préciser la nature des activités conduites en présence d'un enseignant supplémentaire dans la classe permettrait d'avancer dans l'interprétation des résultats.

\section{Conclusion}

Dans l'apprentissage de la lecture-écriture, l'enseignant opère des choix relatifs aux contenus didactiques et aux modalités d'enseignement qu'il souhaite ou est conduit à privilégier. Les marges de manœuvre sont conséquentes et nous avons montré combien les arbitrages opérés influent sur les apprentissages des élèves, et notamment des plus faibles d'entre eux. Nos résultats, tant par leurs limites que par ce qu'ils révèlent, invitent donc à poursuivre l'étude des liens entre les contenus proposés en classe et les performances des élèves.

En effet, si notre étude a mis en évidence quelques repères pour un enseignement efficace de la lecture-écriture (importance du temps consacré à la com-

\section{Bibliographie}

ARONSON J., ZIMMERMAN J. \& CARLOS L. (1998). Improving Student Achievement by Extending School: Is It Just a Matter of Time? Communication au PACE Media/Education Writers Seminar. En ligne : <https://www.wested.org/online_ pubs/po-98-02.pdf> (consulté le 2 avril 2016).

BERLINER D. C. (1990). «What's all the fuss about instructional time? ». In M. Ben-Peretz \& R. Bromme (dir.), The nature of time in schools: Theoretical concepts, practitioner perceptions. New York : Teachers College Press, p.3-35.

BIANCO M. \& BRESSOUX P. (2009). «Effet-classe et effetmaître dans l'enseignement primaire : vers un enseignement efficace de la compréhension? ». In X. Dumay $\&$ V. Dupriez, L'efficacité dans l'enseignement, promesses et zones d'ombre. Bruxelles : De Boeck, p. 35-54.

BIANCO M., PELLENQ C., LAMBERT E., BRESSOUX P., LIMA L. \& DOYEN A.-L. (2012). «Impact of early code-skills and oral-comprehension training on reading achievement in first grade». Journal of Research in Reading, vol.35, n 4 , p. 427-455.

BLATCHFORD P., RUSSELL A. \&WEBSTER R. (2012). Reassessing the impact of teaching assistants: How research challenges practice and policy. New York : Routledge.

BRESSOUX P. (1994). «Les recherches sur les effets-écoles et les effets-maîtres ». Revue française de pédagogie, $n^{\circ} 108$, p. 91-137. préhension, à l'étude de la langue, du temps de présence de l'enseignant par exemple), elle est loin d'être suffisante pour guider les pratiques enseignantes. Comme souligné précédemment, ce n'est pas parce le temps consacré à un domaine particulier semble exercer des effets positifs sur les acquisitions des élèves que toutes les tâches quotidiennes relevant de ce domaine exercent elles-mêmes des effets positifs. II reste donc beaucoup à faire pour préciser ces premières analyses. II s'agira notamment de dépasser la seule problématique du temps d'enseignement pour l'articuler à d'autres, centrées sur les contenus, et progresser dans la description des choix didactiques des enseignants et de leurs effets.

Céline Piquée Univ Rennes, CREAD (EA 3875) celine.piquee@univ-rennes2.fr

Caroline Viriot-Goeldel

Université Paris-Est-Créteil, EA 4384, université Paris 8 , CIRCEFT-ESCOL caroline.viriot-goeldel@u-pec.fr

la recherche en éducation ». Éducation et Didactique, vol.1, $\mathrm{n}^{\circ} 2, \mathrm{p} .73-88$.

BRESSOUX P. (2010). Modélisation statistique appliquée aux sciences sociales. Bruxelles : De Boeck, $2^{\mathrm{e}}$ éd.

BRESSOUX P., AMIGUES R., ARNOUX M., BARRÉ-DE MINIAC C., CLANET J., DESSUS P., HALTE J. F., MAURICE J. J., PERRIN-GLORIAN M. J. \& RABY F. (2002). Les stratégies de l'enseignant en situation d'interaction. Rapport de recherche pour Cognitique, Programme École et Sciences cognitives, ministère de la Recherche.

CARROLL J. B. (1963). «A model of school learning». Teachers College Record, $n^{\circ}$ 64(8), p.723-733.

CHOPIN M.-P. (2010). "Les usages du "temps" dans les recherches sur l'enseignement ». Revue française de pédagogie, n¹70, p.87-110. En ligne : <http://rfp.revues. org/1614> (consulté le 26 septembre 2017).

CONNOR C. M., MORRISON F. J. \& SLOMINSKI L. (2006), "Preschool instruction and children's emergent literacy growth ». Journal of Educational Psychology, vol.98, n 4, p. 665-689.

CONNOR C. M., PIASTA S. B., FISHMAN B., GLASNEY S., SCHATSCHNEIDER C., CROWE E. et al. (2009). «Individualizing student instruction precisely: Effects of child $x$ instruction interactions on first grader's literacy development ». Child Development, vol.80, n¹, p.77-100.

CRAHAY M. (2012). L'école peut-elle être juste et efficace? De 
l'égalité des chances à l'égalité des acquis. Bruxelles: De Boeck.

DEHAENE S. (2013). «Enseigner est une science». Le Monde, 20 décembre 2013. En ligne : <http://www.lemonde.fr/ idees/article/2013/12/20/enseigner-est-unescience_4338294_3232.html> (consulté le 2 avril 2016).

DEAUVIAU J. (2013). Rapport de recherche. Lecture au CP : un effet-manuel considérable. En ligne : <http://www. sciences-sociales.ens.fr/IMG/pdf/rapport_enque_te_lecture_deauvieau.pdf> (consulté le 2 avril 2016).

DELHAXHE A. (1997). «Le temps comme unité d'analyse dans la recherche sur l'enseignement ». Revue française de pédagogie, $\mathrm{n}^{\circ} 118$, p. 106-125.

DENHAM C. \& LIEBERMAN A. (1980). Time to learn. Washington : U.S. Department of Education, National Institute of Education.

DURU-BELLAT M. \& MINGAT A. (1998). «Vérification et falsification dans la recherche en éducation ». In C. Hadji \& J. Baillé, Recherche et éducation. Vers une "nouvelle alliance ». La démarche de preuve en 10 questions. Bruxelles: De Boeck, p.161-190.

ECALLE J. \& MAGNAN A. (2015). L'apprentissage de la lecture et ses difficultés. Paris: Dunod, $2^{\mathrm{e}}$ éd.

GOIGOUX R. (2002). «Tâche et activité en didactique du français : I'apport de la psychologie ergonomique». In J. Dolz, B. Schneuwly, T. Thévenaz-Christen \& M. Wirthner (dir.), Les tâches et leurs entours en classe de français. CD-Rom des Actes du $8^{\mathrm{e}}$ congrès international de la DFLM, organisé à Neuchâtel en septembre 2001 (diffusion AIRDF).

GOIGOUX R. (dir.) (2016). «Étude de l'influence des pratiques d'enseignement de la lecture et de l'écriture sur la qualité des premiers apprentissages ». Rapport de recherche remis à Madame la directrice générale de l'enseignement scolaire (DGESCO-MENESR), ministère de l'Éducation nationale, de I'Enseignement supérieur et de la Recherche En ligne : $<$ http://ife.ens-lyon.fr/ife/recherche/lire-ecrire/rapport/ rapport> (consulté le 3 avril 2016)

GOIGOUX R., JARLÉGAN A. \& PIQUÉE C. (2015). «Évaluer l'influence des pratiques d'enseignement du lire-écrire sur les apprentissages des élèves : enjeux et choix méthodologiques ». Recherches en didactiques. Les Cahiers Théodile, $\mathrm{n}^{\circ} 17, \mathrm{p} .33-52$.

KAMIL M. L., PEARSON D., BIRR MOJE E. \& AFFENBACH P. P. (dir.) (2011). Handbook of reading Research. Volume IV. New York \& Londres : Routledge.

MINGAT A. (1991). «Expliquer la variété des acquisitions au cours préparatoire : les rôles de l'enfant, de la famille et de l'école». Revue française de pédagogie, n`95, p. 47-63.

MINISTÈRE DE L'ÉDUCATION NATIONALE (1999). «L'enseignement de la lecture au CP et au CE1». Dossier, n 106. En ligne : <http://media.education.gouv.fr/file/72/2/4722. pdf> (consulté le 4 avril 2016).

MINISTÈRE DE L'ÉDUCATION NATIONALE (2008). «Programmes d'enseignement de l'école primaire». Bulletin officiel, hors série $\mathrm{n}^{\circ} 3$ du 19 juin 2008.

MINISTÈRE DE L'ÉDUCATION NATIONALE (2015). «Pro- grammes d'enseignement du cycle des apprentissages fondamentaux (cycle 2), du cycle de consolidation (cycle 3) et du cycle des approfondissements (cycle 4) ». Bulletin officiel spécial, $\mathrm{n}^{\circ} 11$ du 26 novembre 2015.

NATIONAL READING PANEL (2000). Report of the National Reading Panel: Teaching children to read: Reports of the subgroups (00-4754). Washington : U.S. Government Printing Office.

NATIONAL INSTITUTES OF CHILDHOOD DEVELOPMENT EARLY CHILDCARE RESEARCH NETWORK NICHD [NICHD] (2005). «Pathways to reading: The role of oral language in the transition to reading ». Developmental Psychology, vol. 41, $n^{\circ} 2$, p. 428-442.

PERRENOUD P. (1984) La fabrication de l'excellence scolaire : du curriculum aux pratiques d'évaluation. Genève : Droz.

PIANTA R. C., LA PARO K. \& HAMRE B. K. (2008). Classroom Assessment Scoring System (CLASS). Baltimore : Paul $\mathrm{H}$. Brookes.

RIOU J. (2017). Étude de l'influence de l'enseignement du code alphabétique sur la qualité des apprentissages des élèves de cours préparatoire. Thèse de doctorat, sciences de l'éducation, université Clermont-Auvergne.

ROSENSHINE B. V. (1980). «How time is spent in elementary classrooms». In C. Denham \& A. Lieberman (dir.), Time to learn. Washington : U.S. Department of Education, National Institute of Education, p.107-126.

SANCHEZ M., MAGNAN A. \& ECALLE J. (2012). «Knowledge about word structure in beginning readers: What specific links are there with word reading and spelling? ». Journal of Psychology of Education, n²7, p. 299-317.

SENSEVY G. (2007). «Caractérisation des pratiques d'enseignement et détermination de leur efficacité. La lecture et les mathématiques au cours préparatoire». Rapport de Recherche pour le Programme incitatif de recherche en éducation et formation (PIREF). Paris : Ministère de la Jeunesse, de l'Éducation et de la Recherche.

SMYTH W. (1985). «Time and school learning». In T. Husén (dir.), International encyclopedia of education. Oxford : Pergamon Press, p. 5265-5272.

SNOW C. E., BURNS M. S. \& GRIFFIN P. (dir.) (1998). Preventing reading difficulties in young children. Washington: National Academies Press.

STORCH S.A. \& WHITEHURST G. J. (2002). «Oral language and code-related precursors to reading: Evidence from a longitudinal structural model ». Developmental Psychology, vol.38, n6, p. 934-947.

SUCHAUT B. (1996). «La gestion du temps à l'école maternelle et primaire : diversité des pratiques et effets sur les acquisitions des élèves». L'année de la recherche en sciences de l'éducation, p.123-153.

SUCHAUT B. BOUGNÈRES A. ET BOUGUEN A. (2014). Sept minutes pour apprendre à lire : à la recherche du temps perdu. En ligne : <https://halshs.archives-ouvertes.fr/ halshs-01062065/> (consulté le 2 avril 2016).

TAYLOR B., PEARSON D., CLARK K. \& WALPOLE S. (2000). «Effective Schools and Accomplished Teachers: Lessons 
about Primary-Grade Reading Instruction in Low-Income Schools». The Elementary School Journal, vol.101, n², p. 121-165.

TAYLOR B., PEARSON D., PETERSON D. \& RODRIGUEZ M.
(2003). «Reading Growth in High-Poverty Classrooms: The Influence of Teacher Practices That Encourage Cognitive Engagement in Literacy Learning ». The Elementary School Journal, vol.104, nº 1, p.3-28.

\section{Annexe}

\section{Typologie des tâches}

\section{PG : Phonographie}

PG1. Étudier les phonèmes (sans écrit)

PG2. Étudier les syllabes orales ou d'autres unités de taille supérieure au phonème (rime)

PG3. Étudier les lettres (noms; différentes écritures; sans valeur sonore)

PG4. Étudier les correspondances entre phonèmes et graphèmes (CGP)

PG5. Étudier la combinatoire ou travailler sur les syllabes

\section{L : Lecture}

L1. Lire silencieusement [M P T]

L2. Reconnaître un mot entier

L3. Déchiffrer un mot

L4. Lire à haute voix [P T]

L5. Écouter la maîtresse/le maître lire à haute voix [P T]

\section{$C$ : Compréhension}

C1. Définir ou expliciter une intention de lecture

C2. Anticiper, formuler ou vérifier des hypothèses

C3. Décrire, commenter une illustration

C4. Expliquer ou reformuler le sens ou évoquer une représentation mentale [P T]

C5. Produire un rappel de récit (complet ou partiel) ou de texte explicatif ou de consigne

C6. Rendre explicite une information implicite

C7. Proposer, débattre ou négocier une interprétation/ des interprétations

C8. Réaliser une tâche écrite impliquant la compréhension (explicite ou implicite) [M P T]
C9. Corriger une tâche écrite portant sur la compréhension [M P T]

\section{E : Écriture}

E1. Calligraphier [L S M]

E2. Copier (avec modèle) [L S M P T]

E3. Copier après disparition du modèle [L S M P T] (copie différée)

E4. Écrire sous la dictée [L S M P T] (le maître décide des unités à écrire)

E5. Produire en combinant des unités linguistiques déjà imprimées [S M P T]

E6. Produire en dictant à autrui [L S M P T]

E7. Produire en encodant soi-même [S M PT] (les élèves choisissent)

E8. Définir, planifier ou organiser la tâche d'écriture (enjeu, destinataire, contenu, plan...)

E9. Revenir sur l'écrit produit : le commenter, le corriger, le réviser, I'améliorer

\section{EL : Étude de la langue}

EL1. Lexique

EL2. Syntaxe

EL3. Morphologie (orthographe des mots, chaîne des accords et désinences verbales)

MD : Mémoire didactique

MD1. Rappeler ou réviser un apprentissage antérieur

MD2. Récapituler un nouvel apprentissage

AUTR : Autre type de tâche (préciser laquelle dans la rubrique «Commentaire»)

0 : Temps mort

Note: $L$ : lettre; $S$ : syllabe; $M$ : mot; $P$ : phrase; $T$ : texte. 


\begin{tabular}{|c|c|c|}
\hline Code & Rubrique & $\begin{array}{l}\text { Exemples de ce que les élèves doivent «faire» (c'est-à-dire agir ou regarder et } \\
\text { écouter d'autres camarades ou l'enseignant agir) }\end{array}$ \\
\hline PG1 & $\begin{array}{l}\text { Étudier les } \\
\text { phonèmes } \\
\text { (sans recours } \\
\text { à l'écriture } \\
\text { alphabétique) }\end{array}$ & $\begin{array}{l}\text { Identifier un «son » (phonème) qui se répète dans une comptine } \\
\text { Proposer des mots comportant un phonème donné } \\
\text { Marquer d'un point ou d'une croix la localisation d'un phonème sous un segment de } \\
\text { droite représentant un mot (ou sous les arcs représentant la suite des syllabes qui com- } \\
\text { posent ce mot oral) } \\
\text { (De manière générale, toutes les tâches qui consistent à manipuler, localiser, segmen- } \\
\text { ter, discriminer, comparer, dénombrer, supprimer, ajouter, permuter, remplacer, fusion- } \\
\text { ner, combiner... des phonèmes) }\end{array}$ \\
\hline PG2 & $\begin{array}{l}\text { Étudier les syl- } \\
\text { labes ou d'autres } \\
\text { unités orales de } \\
\text { taille supérieure } \\
\text { au phonème } \\
\text { (sans recours à } \\
\text { l'écriture } \\
\text { alphabétique) }\end{array}$ & $\begin{array}{l}\text { Frapper des mains en segmentant un mot oral en syllabes } \\
\text { Proposer des mots comportant la syllabe étudiée } \\
\text { Tracer des arcs de cercle représentant les syllabes d'un mot représenté par une image } \\
\text { Proposer des mots qui riment avec un mot cible (le lapin de Sylvain) } \\
\text { Proposer des mots qui commencent par la même attaque qu'un mot cible :TR, CR, } \\
\text { BL... } \\
\text { (De manière générale, toutes les tâches qui consistent à manipuler, localiser, segmen- } \\
\text { ter, discriminer, comparer, dénombrer, supprimer, ajouter, permuter, remplacer, fusion- } \\
\text { ner, combiner... des syllabes, des attaques ou des rimes) }\end{array}$ \\
\hline PG3 & $\begin{array}{l}\text { Étudier les } \\
\text { lettres (noms, } \\
\text { mais pas valeur } \\
\text { sonore; diffé- } \\
\text { rentes écritures; } \\
\text { ordre } \\
\text { alphabétique) }\end{array}$ & $\begin{array}{l}\text { Mémoriser le nom des lettres } \\
\text { Reconnaître des lettres dans les trois principaux systèmes d'écriture (scripte, cursive, } \\
\text { capitales d'imprimerie) } \\
\text { Regrouper des mots qui commencent par la même lettre } \\
\text { Choisir une lettre et demander la lettre qui vient avant ou après } \\
\text { Ordonner trois mots selon l'ordre alphabétique }\end{array}$ \\
\hline PG4 & $\begin{array}{l}\text { Étudier les cor- } \\
\text { respondances } \\
\text { graphophonolo- } \\
\text { giques (P-> G ou } \\
G->P)\end{array}$ & $\begin{array}{l}\text { Étudier la (ou les) valeur(s) sonore(s) d'un graphème } \\
\text { Étudier les différents phonèmes auxquels concoure un graphème } \\
\text { Étudier un graphème muet } \\
\text { Entourer des syllabes écrites comportant le graphème étudié } \\
\text { Proposer un mot comportant un graphème donné } \\
\text { Lister les graphies d'un phonème } \\
\text { Localiser des graphèmes correspondant au phonème étudié } \\
\text { Classer des mots en fonction des CGP } \\
\text { Noter la (ou les) valeur(s) sonore(s) d'un graphème à l'aide de l'alphabet phonétique } \\
\text { international ou d'un archiphonème (écriture prototypique du phonème; le O pour [o]) }\end{array}$ \\
\hline PG5 & $\begin{array}{l}\text { Étudier la } \\
\text { combinatoire }\end{array}$ & $\begin{array}{l}\text { Associer des lettres entre elles pour constituer des syllabes ou des mots } \\
\text { Fusionner des phonèmes pour former des syllabes («coller des sons») } \\
\text { Combiner des syllabes pour former des mots } \\
\text { Mémoriser la valeur sonore de syllabes écrites sans passer par leur décomposition gra- } \\
\text { phémique } \\
\text { Interroger la valeur contextuelle des lettres (banc/banane; homme/chat; [le] vent/ } \\
\text { [elles] roulent) }\end{array}$ \\
\hline
\end{tabular}

\begin{tabular}{l|l|l}
\hline L1 & $\begin{array}{l}\text { Lire silencieuse- } \\
\text { ment [M P T] } \\
\text { Observer un écrit }\end{array}$ & $\begin{array}{l}\text { Repérer (entourer) les mots connus d'une phrase ou d'un paragraphe pour tenter de le } \\
\text { lire («pêche aux mots») } \\
\text { Lire de manière silencieuse et autonome une liste de mots ou une phrase } \\
\text { Prendre connaissance d'une consigne écrite }\end{array}$ \\
\hline L2 & $\begin{array}{l}\text { Reconnaître un } \\
\text { mot entier }\end{array}$ & $\begin{array}{l}\text { Nommer un mot connu, rapidement et sans pouvoir le déchiffrer («mot flash») } \\
\text { Reconnaître visuellement un mot considéré comme une suite ordonnée de lettres, } \\
\text { dans une ou plusieurs écritures («voie directe») } \\
\text { Réviser des mots entiers, appris «globalement» (mots «outils», mots très fréquents...) }\end{array}$ \\
\hline
\end{tabular}




\begin{tabular}{l|l|l}
\hline L3 & $\begin{array}{l}\text { Déchiffrer un } \\
\text { mot (segmenta- } \\
\text { tion, conversion } \\
\text { et fusion) }\end{array}$ & $\begin{array}{l}\text { Décomposer des mots en syllabes ou en graphèmes } \\
\text { Convertir les graphèmes d'un mot en phonèmes } \\
\text { Fusionner ces phonèmes pour reconstituer la valeur sonore de la syllabe puis du mot } \\
\text { Oraliser un mot isolé } \\
\text { Oraliser les mots entourés lors d'une lecture silencieuse et individuelle préalable }\end{array}$ \\
\hline L4 & $\begin{array}{l}\text { Lire à haute voix } \\
{[\mathrm{PT}]}\end{array}$ & $\begin{array}{l}\text { Travailler sur la fluidité de la lecture orale } \\
\text { Lire à haute voix en recréant des unités de sens supérieures aux mots } \\
\text { Écouter un élève relire à haute voix des phrases déjà lues } \\
\text { Suivre des yeux et du doigt une lecture oralisée } \\
\text { Lire aux autres élèves un énoncé qu'ils n'ont pas sous les yeux/écouter cette lecture }\end{array}$ \\
\hline L5 & $\begin{array}{l}\text { Écouter l'ensei- } \\
\text { gnant(e) lire à } \\
\text { haute voix } \\
{[\mathrm{PT}]}\end{array}$ & $\begin{array}{l}\text { Écouter la maîtresse/le maître lire une phrase écrite au tableau } \\
\text { Écouter l'enseignant(e) lire un conte } \\
\text { Écouter un texte enregistré par l'enseignant ou un autre professionnel (acteur) }\end{array}$ \\
\hline
\end{tabular}

\begin{tabular}{|c|c|c|}
\hline EL1 & $\begin{array}{l}\text { Lexique (sens et } \\
\text { construction des } \\
\text { mots) }\end{array}$ & $\begin{array}{l}\text { Lors d'une situation spécifique d'étude de la langue ou durant les situations de lecture ou } \\
\text { d'écriture: } \\
\text { Expliquer le sens d'un mot (nouveau ou polysémique) en contexte } \\
\text { Expliquer un mot à partir d'une image («mot du jour») } \\
\text { Trouver un mot à partir d'une définition } \\
\text { Mimer le sens d'un mot } \\
\text { Trouver des phrases utilisant des homonymes (exemples : vers/vert) } \\
\text { Associer un mot à son contraire (antonyme) } \\
\text { Faire des collections et trouver des mots englobant la collection } \\
\text { Trouver/chasser une image ou un mot intrus par le sens (et non pas intrus par sa gra- } \\
\text { phie par exemple) } \\
\text { Recenser des mots pour parler de quelque chose (de héros, de contes, etc.) } \\
\text { Construire une série en trouvant des mots qui ressemblent à un mot donné (famille de } \\
\text { mots, dérivation) } \\
\text { Réemployer des mots déjà rencontrésS'interroger pour retrouver où l'on a rencontré un } \\
\text { mot (dans une poésie, en français ou dans d'autres disciplines...) }\end{array}$ \\
\hline EL2 & Syntaxe & $\begin{array}{l}\text { Lors d'une situation spécifique d'étude de la langue ou durant les situations de lecture ou } \\
\text { d'écriture: } \\
\text { Repérer les phrases d'un texte, les mots d'une phrase, les groupes de mots d'une } \\
\text { phrase } \\
\text { Différencier ligne et phrase } \\
\text { Mettre en ordre les mots d'une phrase } \\
\text { Faire une phrase syntaxiquement correcte à partir d'une liste de mots } \\
\text { Différencier les phrases syntaxiquement correctes des phrases syntaxiquement incor- } \\
\text { rectes } \\
\text { Dans une phrase, remplacer des mots par d'autres, en ajouter ou en supprimer, et juger } \\
\text { de l'acceptabilité de cette phrase } \\
\text { Repérer des signes de ponctuation (point, virgule, points d'interrogation et d'exclama- } \\
\text { tion, tirets) et comprendre leurs fonctions } \\
\text { Trier, catégoriser (« ranger ensemble») les mots d'une phrase ou d'un texte : mots } \\
\text { outils, verbe, nom, etc. } \\
\text { Faire ou compléter des collections de groupes grammaticaux (déterminant/nom; } \\
\text { déterminant/nom/adjectif; au pluriel, au singulier, au masculin et au féminin) et déga- } \\
\text { ger des règles à partir de ces collections } \\
\text { Associer un pronom personnel et le nom qu'il remplace } \\
\text { Étudier des mots outils fréquents (pronoms personnels, déterminants, prépositions) et } \\
\text { questionner leur valeur : faire percevoir la signification d'un mot outil par opposition à } \\
\text { un autre (ex. un bateau/des bateaux) }\end{array}$ \\
\hline
\end{tabular}




\begin{tabular}{|c|c|c|}
\hline EL3 & $\begin{array}{l}\text { Morphologie } \\
\text { (orthographe des } \\
\text { mots, chaîne des } \\
\text { accords et dési- } \\
\text { nences verbales) }\end{array}$ & $\begin{array}{l}\text { Lors d'une situation spécifique d'étude de la langue ou durant les situations de lecture ou } \\
\text { d'écriture: } \\
\text { Observer l'orthographe de mots variables ou invariables } \\
\text { Mémoriser l'orthographe de mots fréquents déjà rencontrés } \\
\text { Identifier/orthographier les marques grammaticales : singulier/pluriel; masculin/fémi- } \\
\text { nin } \\
\text { Étudier les formes verbales (temps, genre et nombre) } \\
\text { S'interroger sur l'orthographe de mots contenant des lettres muettes lexicales (chat) } \\
\text { Observer et formuler une régularité grammaticale }\end{array}$ \\
\hline E1 & $\begin{array}{l}\text { Calligraphier } \\
\text { [L S M] }\end{array}$ & $\begin{array}{l}\text { S'exercer à tracer les lettres en cursive et à les attacher (sur l'ardoise ou le cahier) } \\
\text { Reproduire avec son doigt le tracé d'une lettre } \\
\text { Apprendre le tracé des majuscules }\end{array}$ \\
\hline E2 & $\begin{array}{l}\text { Copier } \\
\text { (avec modèle) } \\
{[\text { L S M P T] }}\end{array}$ & $\begin{array}{l}\text { Copier une syllabe ou un mot sur un cahier ou sur une ardoise d'après un modèle pré- } \\
\text { sent } \\
\text { Compléter à l'écrit des mots à l'aide d'une ou de plusieurs syllabes données avec la } \\
\text { consigne } \\
\text { Écrire une phrase en recopiant des mots donnés sur des étiquettes } \\
\text { Écrire une poésie apprise en la recopiant d'une page de manuel }\end{array}$ \\
\hline E3 & $\begin{array}{l}\text { Copier après dis- } \\
\text { parition du } \\
\text { modèle (copie } \\
\text { différée) } \\
\text { [L S M P T] }\end{array}$ & $\begin{array}{l}\text { Copier sur le verso d'une feuille un mot ou une phrase écrit(e) sur le recto } \\
\text { Copier sur son ardoise une lettre puis une syllabe écrite au tableau puis cachée } \\
\text { Reproduire une courte phrase écrite puis effacée du tableau, après observation et } \\
\text { mémorisation }\end{array}$ \\
\hline E4 & $\begin{array}{l}\text { Écrire sous la } \\
\text { dictée } \\
\text { [L S M P T] } \\
\text { (le maître décide } \\
\text { des unités à } \\
\text { écrire) }\end{array}$ & $\begin{array}{l}\text { Écrire sous la dictée du maître une liste de syllabes } \\
\text { Écrire une courte phrase dictée } \\
\text { Écrire sur l'ardoise un mot dicté }\end{array}$ \\
\hline E5 & $\begin{array}{l}\text { Produire en com- } \\
\text { binant des unités } \\
\text { linguistiques déjà } \\
\text { imprimées } \\
\text { [S M P] }\end{array}$ & $\begin{array}{l}\text { Fabriquer des mots en assemblant des syllabes pré-imprimées } \\
\text { Fabriquer une phrase en assemblant des étiquettes-mots }\end{array}$ \\
\hline E6 & $\begin{array}{l}\text { Produire en dic- } \\
\text { tant à autrui } \\
{[\text { L S M P] }}\end{array}$ & $\begin{array}{l}\text { Dicter à l'enseignant ou à autrui une lettre pour les correspondants } \\
\text { Dicter la date ou une consigne à l'enseignant (dictée à l'adulte) } \\
\text { Écrire une liste de prénoms en les dictant à l'enseignant } \\
\text { Dicter à l'enseignant la suite d'une histoire lue }\end{array}$ \\
\hline E7 & $\begin{array}{l}\text { Produire en } \\
\text { encodant soi- } \\
\text { même } \\
\text { [S M P T] } \\
\text { (les élèves } \\
\text { choisissent) }\end{array}$ & $\begin{array}{l}\text { Écrire une devinette } \\
\text { Écrire une lettre (à un destinataire précis) en comblant les trous } \\
\text { Préparer une affiche pour les élections (c'est-à-dire «pourquoi vous devez voter pour } \\
\text { moi ») } \\
\text { Écrire une légende sous une image } \\
\text { Écrire la fin d'une phrase commencée par l'enseignant } \\
\text { Compléter des mots en écrivant (sans modèle) la syllabe manquante }\end{array}$ \\
\hline E8 & $\begin{array}{l}\text { Définir, planifier } \\
\text { ou organiser la } \\
\text { tâche d'écriture } \\
\text { (enjeu, destina- } \\
\text { taire, contenu, } \\
\text { plan, } \\
\text { contrainte(s) à } \\
\text { respecter...) }\end{array}$ & $\begin{array}{l}\text { Se demander à quoi ressemble le type d'écrit à produire compte tenu du projet de } \\
\text { classe } \\
\text { Faire la liste de ce que les élèves veulent aborder dans leur texte } \\
\text { Rappeler les supports sur lesquels les élèves peuvent s'appuyer pour écrire un mot } \\
\text { Faire la liste d'étapes et de règles nécessaires pour écrire } \\
\text { Indiquer l'orthographe de mots utilisables pour écrire }\end{array}$ \\
\hline
\end{tabular}




\begin{tabular}{|c|c|c|}
\hline E9 & $\begin{array}{l}\text { Revenir sur l'écrit } \\
\text { produit : le com- } \\
\text { menter, le corri- } \\
\text { ger, le réviser, } \\
\text { l'améliorer }\end{array}$ & $\begin{array}{l}\text { Commenter l'écrit produit } \\
\text { Comparer sa production avec le modèle ou un autre écrit } \\
\text { Modifier son écrit en tenant compte des corrections apportées par le maître } \\
\text { Relire son écriture de mots ou de phrases ou celle d'un pair }\end{array}$ \\
\hline C1 & $\begin{array}{l}\text { Définir ou expli- } \\
\text { citer une inten- } \\
\text { tion de lecture }\end{array}$ & $\begin{array}{l}\text { Avant de commencer à lire : expliquer le but de la tâche ou de l'activité à venir } \\
\text { (aujourd'hui, on va...) } \\
\text { En cours de lecture ou à la fin : revenir sur le but de l'activité } \\
\text { Amener à adapter la lecture selon le type d'écrit }\end{array}$ \\
\hline C2 & $\begin{array}{l}\text { Anticiper, formu- } \\
\text { ler ou vérifier } \\
\text { des hypothèses }\end{array}$ & $\begin{array}{l}\text { Avant la lecture, dire ce que l'on s'attend à découvrir dans la lecture en prenant appui } \\
\text { sur certains éléments (titres, certaines illustrations, } 4^{\text {e }} \text { de couverture, les connaissances } \\
\text { des élèves, etc.); accueillir la réaction des élèves (dimension psychoaffective) } \\
\text { En cours de lecture, formuler des hypothèses sur la suite, les vérifier en avançant dans } \\
\text { la lecture } \\
\text { À la fin de la lecture, comparer les hypothèses de départ avec ce qui a été lu et de dis- } \\
\text { cuter des divergences } \\
\text { À la fin de la lecture, synthétiser les hypothèses données et rappeler celles qui ont été } \\
\text { validées lors de la lecture } \\
\text { Convoquer des connaissances sur le monde } \\
\text { Convoquer des lectures ou souvenirs de lecture ou d'expériences personnelles }\end{array}$ \\
\hline C3 & $\begin{array}{l}\text { Décrire, com- } \\
\text { menter une } \\
\text { illustration }\end{array}$ & $\begin{array}{l}\text { Observer l'illustration (avant, pendant ou après la lecture) pour conforter ou infléchir le } \\
\text { sens qu'on est en train d'élaborer } \\
\text { Décrire l'illustration (avant, pendant ou après la lecture) } \\
\text { Comparer les illustrations avec le texte (redondance, ajouts d'informations, informa- } \\
\text { tions contradictoires...) } \\
\text { Fabriquer une illustration du passage qui vient d'être lu ou de la totalité du texte }\end{array}$ \\
\hline C4 & $\begin{array}{l}\text { Expliquer ou } \\
\text { reformuler le } \\
\text { sens } \\
{[\mathrm{PT}]}\end{array}$ & $\begin{array}{l}\text { Répondre oralement à des questions portant sur les informations figurant dans le texte } \\
\text { (explicite) } \\
\text { Paraphraser ou reformuler la phrase ou le texte dans une langue plus accessible } \\
\text { Apporter des explications pour rendre compréhensible un événement, une action, un } \\
\text { phénomène, les caractéristiques d'un personnage tels qu'ils figurent dans le texte } \\
\text { Inciter les élèves à se représenter une scène ou des événements : «se faire le film » } \\
\text { (représentation mentale) } \\
\text { Rapprocher le texte d'un autre texte (ou contexte, ou élément de la vie quotidienne) } \\
\text { de sorte que les élèves puissent mobiliser des savoirs qu'ils possèdent déjà pour com- } \\
\text { prendre le texte }\end{array}$ \\
\hline C5 & $\begin{array}{l}\text { Produire un rap- } \\
\text { pel de récit } \\
\text { (complet ou } \\
\text { partiel) }\end{array}$ & $\begin{array}{l}\text { «Dire où on en est», raconter le début du texte qu'on est en train de lire } \\
\text { Raconter tout le texte à la fin du travail } \\
\text { Dire «tout ce que l'on a appris sur» un personnage, l'organisation temporelle, les lieux } \\
\text { de l'intrigue, etc. } \\
\text { Élaborer une fiche sur un personnage, sur les déplacements, la suite chronologique des } \\
\text { événements... } \\
\text { Jouer l'histoire (théâtre, mimes, marionnettes...) }\end{array}$ \\
\hline C6 & $\begin{array}{l}\text { Rendre explicite } \\
\text { une information } \\
\text { implicite }\end{array}$ & $\begin{array}{l}\text { Rechercher des indices et mettre en relation des éléments disjoints (à votre avis, pour- } \\
\text { quoi? Comment?) } \\
\text { Inférer les sentiments et états mentaux des personnages (c'est-à-dire ce que le person- } \\
\text { nage veut, ressent, sait ou croit à ce moment-là de l'histoire), lorsqu'ils ne figurent pas } \\
\text { tels que dans le texte }\end{array}$ \\
\hline C7 & $\begin{array}{l}\text { Proposer, } \\
\text { débattre ou } \\
\text { négocier une } \\
\text { interprétation }\end{array}$ & $\begin{array}{l}\text { Discuter les différentes interprétations émises lors de la lecture d'un passage dont le } \\
\text { sens pose problème } \\
\text { Jouer une scène pour déterminer ce qui est possible et ce qui ne l'est pas parmi les dif- } \\
\text { férentes interprétations } \\
\text { Participer à un débat interprétatif }\end{array}$ \\
\hline
\end{tabular}




\begin{tabular}{l|l|l}
\hline C8 & $\begin{array}{l}\text { Réaliser une } \\
\text { tâche écrite } \\
\text { impliquant la } \\
\text { compréhension } \\
\text { [M P T] }\end{array}$ & $\begin{array}{l}\text { Répondre à des questions du type : « vrai/faux» } \\
\text { Répondre par écrit à un questionnaire de compréhension } \\
\text { Compléter le texte (ou une reformulation) qui comporte des trous (des blancs) } \\
\text { Choisir/entourer la bonne illustration } \\
\text { Réaliser une illustration en s'attachant à rendre compte du passage lu } \\
\text { Proposer ou compléter un classement d'images ou d'extraits de texte dans l'ordre } \\
\text { chronologique }\end{array}$ \\
\hline C9 & $\begin{array}{l}\text { Corriger une } \\
\text { tâche écrite por- } \\
\text { tant sur la com- } \\
\text { préhension } \\
\text { [M P T] }\end{array}$ & $\begin{array}{l}\text { Donner et justifier des réponses soit en recourant au texte soit en se servant de ses } \\
\text { connaissances } \\
\text { Recopier la réponse exacte énoncée par l'enseignant(e) } \\
\text { Modifier ses réponses écrites, si nécessaire, en s'attachant à rendre compte de sa com- } \\
\text { préhension de la phrase ou du texte lu ou écouté }\end{array}$ \\
\hline
\end{tabular}

Tableau 1. Décomposition de la variance des scores de fin de CP

\begin{tabular}{|c|c|c|c|}
\hline Paramètres & Modèle vide & Modèle 1 & Modèle 2 \\
\hline \multicolumn{4}{|l|}{ Effets fixes } \\
\hline Constante & $-0,007(0,04)$ & $0,03(0,05)^{* * *}$ & $-0,09(0,16)$ \\
\hline Score individuel initial & & $0,71(0,02)^{* * *}$ & $0,71(0,02)^{* * *}$ \\
\hline Fille (référence garçon) & & $0,08(0,03)^{* * *}$ & $0,08(0,03)^{* * *}$ \\
\hline \multicolumn{4}{|l|}{ Profession des parents : } \\
\hline Catégorie défavorisée & & $-0,12(0,04)^{* * *}$ & $-0,11(0,04)^{* * *}$ \\
\hline $\begin{array}{l}\text { Catégorie intermédiaire (référence catégorie } \\
\text { favorisée) } \\
\text { Âge : }\end{array}$ & & $-0,03(0,04)$ & $-0,03(0,04)$ \\
\hline $\begin{array}{l}\text { Né avant } 2007 \\
\text { (retard scolaire) }\end{array}$ & & $-0,61(0,07)^{* * *}$ & $-0,61(0,07)^{* * *}$ \\
\hline Né entre mai et août 2007 & & $-0,02(0,03)$ & $-0,02(0,03)$ \\
\hline $\begin{array}{l}\text { Né entre sept. et déc. } 2007 \text { (référence né entre } \\
\text { janv. et avril } 2007 \text { ou en avance) }\end{array}$ & & $-0,02(0,03)$ & $-0,02(0,03)$ \\
\hline \multicolumn{4}{|l|}{ Langue parlée à la maison : } \\
\hline Autre & & $0,04(0,06)$ & $0,03(0,06)$ \\
\hline Français + autre (référence français) & & $0,12(0,04)^{* * *}$ & $0,12(0,04)^{* * *}$ \\
\hline Semaine de 4 jours (référence 4,5 jours) & & & $-0,08(0,07)$ \\
\hline Éducation prioritaire & & & $-0,03(0,07)$ \\
\hline Niveau moyen initial & & & $-0,19(0,08)^{* *}$ \\
\hline Hétéro moyenne initial & & & $0,05(0,16)$ \\
\hline \% d'élèves de milieu favorisé & & & $0,24(0,19)$ \\
\hline Ancienneté au CP & & & $0,01(0,01)$ \\
\hline \multicolumn{4}{|l|}{ Effets aléatoires } \\
\hline Niveau 2 : var. interclasses & $0,1255(0,02)$ & $0,0807(0,01)$ & $0,0735(0,01)$ \\
\hline Niveau 1 : var. intraclasse & $0,8703(0,03)$ & $0,3898(0,01)$ & $0,3898(0,01)$ \\
\hline$-2 \log L$ & 6867,191 & 4910,292 & 4900,814 \\
\hline
\end{tabular}

Notes : $\mathrm{N}=2$ 481. Seuil de significativité des effets fixes : ${ }^{*}$ : significatif au seuil de $10 \%$; ${ }^{* *}$ : significatif au seuil de $5 \%$; ${ }^{* * *}$ : significatif au seuil de $1 \%$. Les erreurs type des coefficients figurent entre parenthèses. 
Tableau 5. Effets du temps passé sur chaque domaine de tâches sur le score global (présentation synthétique)

\begin{tabular}{|c|c|c|c|c|c|}
\hline Paramètres & Modèle 1 & Modèle 2 & Modèle 3 & Modèle 4 & Modèle 5 \\
\hline \multicolumn{6}{|l|}{ Effets fixes } \\
\hline ZPG & $0,01(0,03)$ & & & & \\
\hline ZL & & $0,001(0,03)$ & & & \\
\hline ZEL & & & $0,11(0,03)^{* * *}$ & & \\
\hline ZE & & & & $0,02(0,03)$ & \\
\hline ZC & & & & & $0,03(0,03)$ \\
\hline Effets aléatoires & & & & & \\
\hline $\begin{array}{l}\text { Niveau } 2 \text { : var. } \\
\text { interclasses }\end{array}$ & $0,0733(0,01)$ & $0,0734(0,01)$ & $0,0631(0,01)$ & $0,0731(0,01)$ & $0,0725(0,01)$ \\
\hline $\begin{array}{l}\text { Niveau } 1 \text { : var. } \\
\text { intraclasse }\end{array}$ & $0,3898(0,01)$ & $0,3898(0,01)$ & $0,3898(0,01)$ & $0,3898(0,01)$ & $0,3898(0,01)$ \\
\hline$-2 \log L$ & 4900,599 & 4900,655 & 48885,842 & 4900,238 & 4899,370 \\
\hline
\end{tabular}

Notes : $\mathrm{N}=2$ 481. Seuil de significativité des effets fixes : * : significatif au seuil de $10 \%$; ${ }^{*}$ : significatif au seuil de $5 \%$; **** significatif au seuil de $1 \%$. Les erreurs type des coefficients figurent entre parenthèses.

Tableau 6. Non-linéarité et effet différencié du temps consacré à l'étude de la langue et à la compréhension sur le score global (présentation synthétique)

\begin{tabular}{l|c|c|c}
\hline Paramètres & Modèle 1 & Modèle 2 & Modèle 3 \\
\hline Effets fixes & $0,08(0,03)^{* *}$ & $0,04(0,03)$ & \\
ZC & $-0,04(0,02)^{* *}$ & $-0,17(0,05)^{* * *}$ & $0,11(0,03)^{* * *}$ \\
ZC* ZC & & & $-0,04(0,01)^{* * *}$ \\
ZC* niveau moyen initial & & & $0,0620(0,01)$ \\
ZEL & & $0,0658(0,01)$ & $0,3885(0,01)$ \\
ZEL*score individuel initial & $0,0690(0,01)$ & $0,3898(0,01)$ & 4875,477 \\
Effets aléatoires & $0,3898(0,01)$ & 4892,470 & \\
Niveau 2: var. interclasses & 4894,358 & & \\
Niveau 1 : var. intraclasse & & & \\
\hline - 2 log L & & & \\
\hline
\end{tabular}

Notes: $\mathrm{N}=2481$. Seuil de significativité des effets fixes : ${ }^{*}$ : significatif au seuil de $10 \%$; ** : significatif au seuil de $5 \%$;

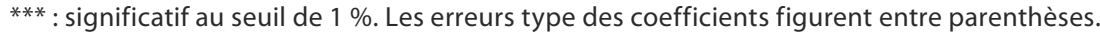


Tableau 9. Effets du temps passé sur chaque domaine de tâches en présence du maître sur le score global (présentation synthétique)

\begin{tabular}{l|c|c|c|c|c}
\hline Paramètres & Modèle 1 & Modèle 2 & Modèle 3 & Modèle 4 & Modèle 5 \\
\hline $\begin{array}{l}\text { Effets fixes } \\
\text { Ztpspg }\end{array}$ & $0,02(0,03)$ & & & & \\
Ztpsl & & $0,01(0,03)$ & & \\
Ztpsel & & & $0,11(0,03) * * *$ & $0,03(0,03)$ & $0,04(0,03)$ \\
Ztpse & & & & \\
Ztpsc & & & & \\
Effets aléatoires & & & & \\
Niveau 2: var. interclasses & $0,0731(0,01)$ & $0,0734(0,01)$ & $0,0615(0,01)$ & $0,0727(0,01)$ & $0,0718(0,01)$ \\
Niveau 1 : var. intraclasse & $0,3898(0,01)$ & $0,3898(0,01)$ & $0,3898(0,01)$ & $0,3898(0,01)$ & $0,3898(0,01)$ \\
\hline-2 log L & 4900,355 & 4900,729 & 48883,516 & 4899,631 & 4936,378 \\
\hline
\end{tabular}

Notes : $\mathrm{N}=2481$. Seuil de significativité des effets fixes : ${ }^{*}$ : significatif au seuil de $10 \%$; ${ }^{* *}$ : significatif au seuil de $5 \%$; *** : significatif au seuil de $1 \%$. Les erreurs type des coefficients figurent entre parenthèses.

Tableau 10. Effets différenciés et non-linéarités du temps passé sur chaque domaine de tâches en présence du maître sur le score global (présentation synthétique)

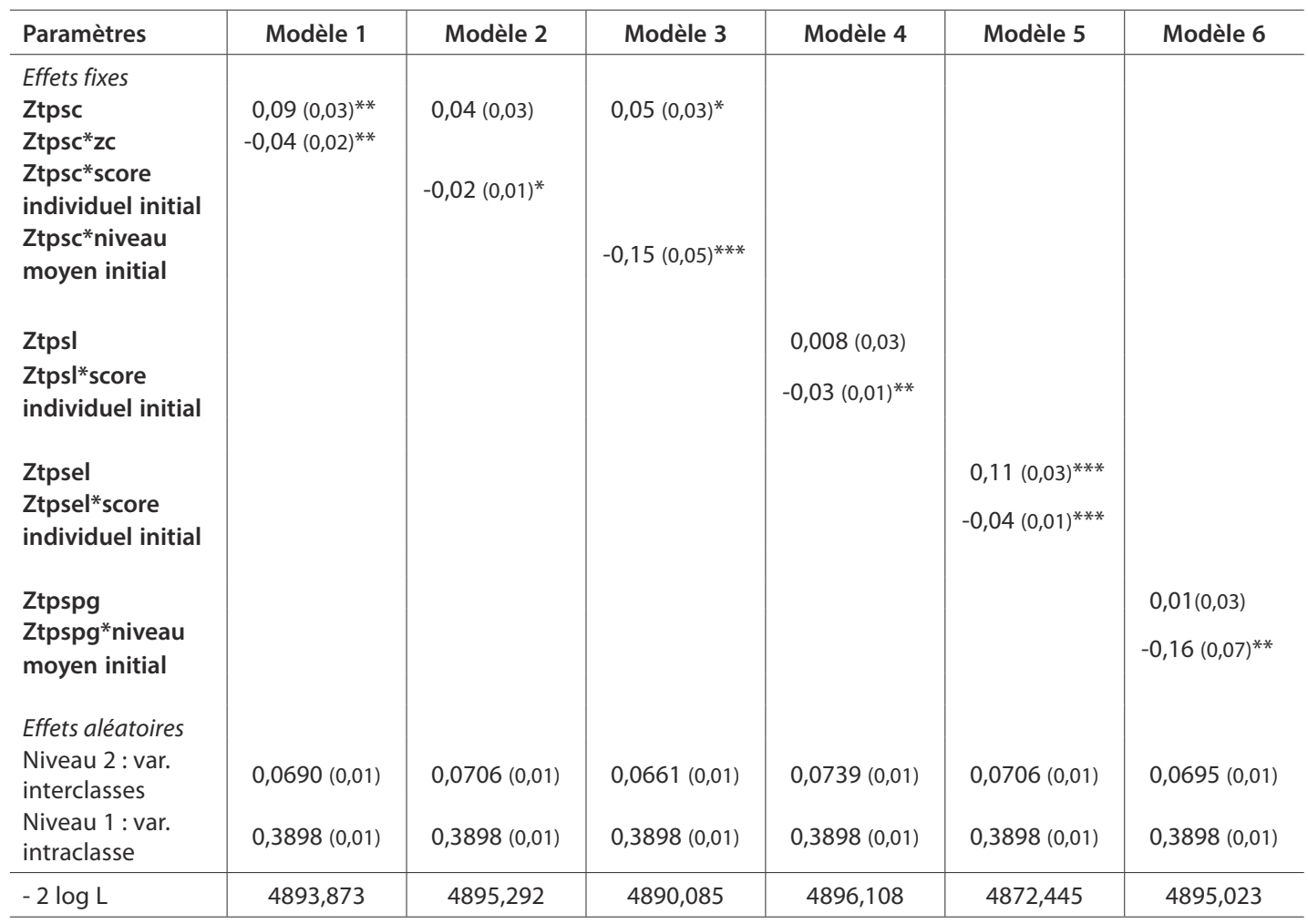

Notes : $\mathrm{N}=2481$. Seuil de significativité des effets fixes : * : significatif au seuil de $10 \%$; ${ }^{* *}$ : significatif au seuil de $5 \%$; $*^{* * *}$ : significatif au seuil de $1 \%$. Les erreurs type des coefficients figurent entre parenthèses. 
Tableau 11. Effets différenciés du temps passé sur chaque domaine de tâches en dehors de la présence du maître sur le score global (présentation synthétique)

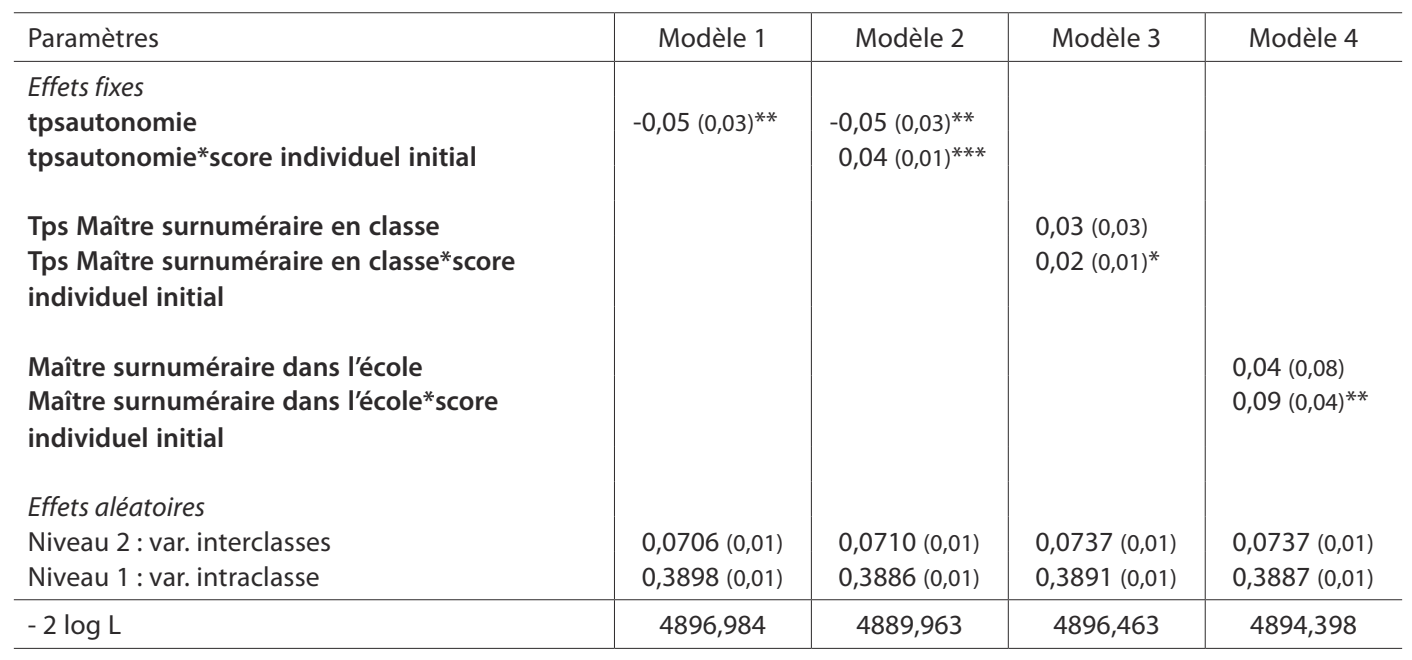

Notes : $\mathrm{N}=2$ 481. Seuil de significativité des effets fixes : ${ }^{*}$ : significatif au seuil de $10 \%$; ${ }^{* *}$ : significatif au seuil de $5 \%$; *** : significatif au seuil de $1 \%$. Les erreurs type des coefficients figurent entre parenthèses 